\title{
Supramolecular synthon pattern in solid clioquinol and cloxiquine (APIs of antibacterial, antifungal, antiaging and antituberculosis drugs) studied by ${ }^{35} \mathrm{Cl} \mathrm{NQR},{ }^{1} \mathrm{H}-{ }^{17} \mathrm{O}$ and ${ }^{1} \mathrm{H}-{ }^{14} \mathrm{~N}$ NQDR and DFT/QTAIM
}

\author{
Jolanta Natalia Latosińska • Magdalena Latosińska • \\ Marzena Agnieszka Tomczak • Janez Seliger • \\ Veselko Žagar
}

Received: 18 April 2010 /Accepted: 11 October 2010 /Published online: 16 November 2010

(C) The Author(s) 2010. This article is published with open access at Springerlink.com

\begin{abstract}
The quinolinol derivatives clioquinol (5-chloro7-iodo-8-quinolinol, Quinoform) and cloxiquine (5-chloro8-quinolinol) were studied experimentally in the solid state via ${ }^{35} \mathrm{Cl} \mathrm{NQR},{ }^{1} \mathrm{H}-{ }^{17} \mathrm{O}$ and ${ }^{1} \mathrm{H}-{ }^{14} \mathrm{~N}$ NQDR spectroscopies, and theoretically by density functional theory (DFT). The supramolecular synthon pattern of $\mathrm{O}-\mathrm{H} \cdots \mathrm{N}$ hydrogen bonds linking dimers and $\pi-\pi$ stacking interactions were described within the QTAIM (quantum theory of atoms in molecules) /DFT (density functional theory) formalism. Both proton donor and acceptor sites in $\mathrm{O}-\mathrm{H} \cdots \mathrm{N}$ bonds were characterized using ${ }^{1} \mathrm{H}^{17}{ }^{17} \mathrm{O}$ and ${ }^{1} \mathrm{H}^{1}{ }^{14} \mathrm{~N}$ NQDR spectroscopies and QTAIM. The possibility of the existence of $\mathrm{O}-\mathrm{H} \cdots \mathrm{H}-\mathrm{O}$ dihydrogen bonds was excluded. The weak intermolecular interactions in the crystals of clioquinol and cloxiquine were detected and examined. The results obtained in this work suggest that considerable differences in the NQR parameters for the planar and twisted supramolecular synthons permit differentiation between specific polymorphic forms, and indicate that the more planar supramolecular synthons are accompanied by a
\end{abstract}

J. N. Latosińska $(\bowtie) \cdot$ M. Latosińska $\cdot$ M. A. Tomczak

Faculty of Physics, Adam Mickiewicz University,

Umultowska 85,

61-614 Poznań, Poland

e-mail: Jolanta.Latosinska@amu.edu.pl

J. Seliger $\cdot$ V. Žagar

"Jozef Stefan" Institute,

Jamova 39,

1000 Ljubljana, Slovenia

J. Seliger

Faculty of Mathematics and Physics, University of Ljubljana,

Jadranska 19,

1000 Ljubljana, Slovenia greater number of weaker hydrogen bonds linking them and stronger $\pi \cdots \pi$ stacking interactions.

Keywords Clioquinol $\cdot$ Cloxiquine $\cdot$ Nuclear quadrupole resonance - Antiaging - Anticancer - Antituberculosis .

Antibacterial · Antifungal · DFT · QTAIM · Intermolecular interactions · Supramolecular synthon · Polymorphism

\section{Introduction}

The quinolinol derivatives clioquinol (5-chloro-7-iodo-8quinolinol, Quinoform, Quinambicide, Vioform) and cloxiquine (5-chloro-8-quinolinol, Chloroxychinolin, Cloxiquine, Dermofongin A) Fig. 1 are active pharmaceutical ingredients (APIs) of a wide spectrum of known potent antibacterial, antifungal and antiamoebic agents used in the treatment of dermatoses [1-3] and antiseptic or disinfectant formulations [4] that have been known for decades. Both are members of the group of drugs called 8-quinolinols which inhibit DNA replication and are active against both viral and protozoal infections [5]. Clioquinol is also used to treat diarrhea and other gastrointestinal disorders, skin infections such as eczema, athlete's foot, jock itch, ringworm, and some bacteria (Staphylococcus, Streptococcus, E. coli), yeasts (Candida albicans), and some protozoan parasites (particularly Trichomonas sp. [6, 7]), and recently tuberculosis [8]. On the other hand, both clioquinol and cloxiquine are common causes of epigastric discomfort, contact dermatitis and neuropathy, and both are considered mutagens. Clioquinol produces not only allergic reactions, eosinophilia and hyperthyreosis, but it also exhibits teratogenic and carcinogenic effects, and is the 
Fig. 1 The molecular structures of 8-quinolinol derivatives $(\mathrm{R}=\mathrm{I}$ in clioquinol, $\mathrm{R}=\mathrm{H}$ in cloxiquine)

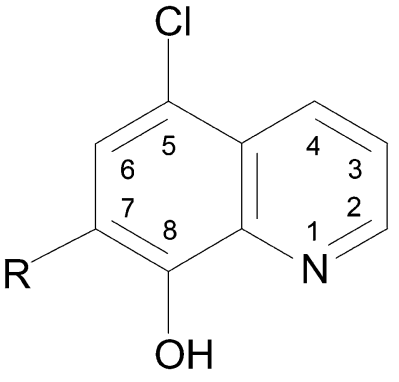

most toxic of the antibacterial compounds which can depress the central nervous system. It was withdrawn from use in the 1970s due to serious adverse events like blindness, paralysis or death [9]. The increasing interest in both compounds stems from the recent finding that cloxiquine exhibits good antituberculosis activity, even for multidrug resistant (MDR) isolates [10], whereas clioquinol, which is known to be extremely neurotoxic (on account of its ability to chelate metals [11]) in large doses, and is one of the agents that leads to lethal subacute myelo-optico-neuropathy (SMON) $[12,13]$ in small doses, has been found to be capable of reversing the progression of neurodegenerative disorders. The latter effect is probably due to the action directed at the protein called CLK-1 ("clock-1"), and thus helps to suppress the initiation of Alzheimer's, Parkinson's and Huntington's diseases by slowing aging [14-17] due to its free radical scavenging capabilities. Very recently, clioquinol was found to inhibit proteasomes, display preclinical activity in leukemia and myeloma [18], and to exert anticancer effects both in vitro and in vivo [19]. Unfortunately, the mechanism of its action has not been identified yet, although it is believed to be related to the iodine content.

Considering the above discussion, and the differences in their biological activities, a comparison of the structural and electronic properties of clioquinol and cloxiquine (which differs from clioquinol only in its lack of iodine at the 7 position of the quinoline ring) appears to be a very promising line of research, and therefore deserves to be the focus of detailed studies. It is known that the biological activities of compounds are related to their chemical structures, especially their electron density distributions and bonding capabilities [20,21]. Ever since the first experiments in solid-state nuclear quadrupole resonance (NQR), the great potential of this molecule-specific methodwhich allows the nondestructive characterization of solid pharmaceutical products - for the analysis of biological systems has been recognized [22-25]. The electric field gradient (EFG) tensor depends on the positions and charges of the nuclei and electrons about the quadrupolar nucleus, and thus the quadrupole coupling constant-which is the largest (in absolute value) principal component of the EFG tensor multiplied by the nuclear quadrupole moment and divided by Planck's constant-reflects the electron distribution in the vicinity of the quadrupolar nuclei, making it a very sensitive tool for investigating molecular and crystal structure in detail. NQR appears to be the optimal method for studying clioquinol and cloxiquine, because-rather unusually - both compounds contain three kinds of quadrupolar nuclei in their molecules: ${ }^{14} \mathrm{~N},{ }^{17} \mathrm{O}$ and ${ }^{35} \mathrm{Cl}$ (indeed, clioquinol actually contains four, including ${ }^{127} \mathrm{I}$ ). In order to elucidate these details and to aid our understanding of the differences in the biological activities of both compounds, we performed a joint study that applied NQR for different isotopes $\left({ }^{14} \mathrm{~N},{ }^{17} \mathrm{O}\right.$ and $\left.{ }^{35} \mathrm{Cl}\right)$, as well as density functional theory (DFT), which reveals the local and global electron density distribution in the molecules. We expect that this combined study will permit a detailed understanding of the differences in the structural features of clioquinol and cloxiquine, and contribute to an explanation of the role of clioquinol at the molecular level, especially the functional implications of iodine substitution and $\mathrm{O}-\mathrm{H} \cdot \cdots \mathrm{N}$ hydrogen bonding formation for the recognition and binding of clioquinol molecules to the mitochondrial enzyme CLK-1 (also known as COQ7).

\section{Experimental}

High-purity polycrystalline samples of clioquinol and cloxiquine (95\% and 97\%, respectively) were purchased from Sigma-Aldrich and used without any additional purification.

\section{NQR spectroscopy}

The ${ }^{35} \mathrm{Cl},{ }^{17} \mathrm{O}$ and ${ }^{14} \mathrm{~N}$ nuclei have spins $I=3 / 2,5 / 2$ and 1 , respectively, and therefore, when there is no external magnetic field, ${ }^{35} \mathrm{Cl}$ exhibits two doubly degenerate, ${ }^{17} \mathrm{O}$ exhibits three doubly degenerate, and ${ }^{14} \mathrm{~N}$ exhibits nondegenerate nuclear quadrupole energy levels. Their energies depend on the nuclear quadrupole moment $e Q$ and on the electric field gradient (EFG) tensor $\mathrm{V}_{i k}=\partial^{2} \mathrm{~V} / \partial \mathrm{x}_{i} \partial \mathrm{x}_{k}$, which consists of the second derivatives of the electrostatic potential $V$ with respect to coordinates originating at the position of the nucleus. The symmetric traceless secondrank $\mathrm{EFG}$ tensor has three principal values: $\mathrm{V}_{\mathrm{ZZ}}=e q, \mathrm{~V}_{\mathrm{YY}}$ and $\mathrm{V}_{\mathrm{XX}}\left(\left|\mathrm{V}_{\mathrm{ZZ}}\right|<>\left|\mathrm{V}_{\mathrm{YY}}\right| \geq\left|\mathrm{V}_{\mathrm{XX}}\right|\right)$, which are used to obtain two unique NQR parameters: the nuclear quadrupole coupling constant $\left(\mathrm{e}^{2} \mathrm{Qqh}^{-1}\right)$ and the asymmetry parameter $(\eta)$, which are related to the NQR frequencies $(\nu)$ through the following equations [26]:

a) $\mathrm{For}{ }^{35} \mathrm{Cl}$, the frequencies do not uniquely depend on the quadrupole coupling constant $\mathrm{e}^{2} \mathrm{qQ} / \mathrm{h}$ and the asymmetry parameter $\eta$ :

$$
v\left({ }^{35} \mathrm{Cl}\right)=\frac{\mathrm{e}^{2} \mathrm{Qq}}{2 \mathrm{~h}} \sqrt{1+\eta^{2} / 3},
$$

but for biologically active compounds $\eta<<0.1$ 
b) For ${ }^{17} \mathrm{O}$, the three NQR frequencies, which are usually termed $v_{5 / 2-1 / 2}>v_{5 / 2-3 / 2} \geq v_{3 / 2-1 / 2}$, uniquely depend on $\mathrm{e}^{2} \mathrm{qQ} / \mathrm{h}$ and $\eta$, but can be calculated via the following secular equation:

$x^{3}-7\left(3+\eta^{2}\right) x-20\left(1-\eta^{2}\right)=0$,

where $x$ is a solution of Eq. 2, and the energies of the $\mathrm{NQR}$ levels can be calculated using the formula $E=$ $\left(\mathrm{e}^{2} \mathrm{qQh}^{-1} / 20\right) x$

c) For ${ }^{14} \mathrm{~N}$, the three NQR frequencies are usually termed $v_{+} v_{-} \geq v_{0}$ and uniquely depend (in a similar manner to those for ${ }^{17} \mathrm{O}$ ) on the quadrupole coupling constant $\mathrm{e}^{2} \mathrm{qQ} / \mathrm{h}$ and the asymmetry parameter $\eta$ :

$$
\begin{array}{r}
v_{+}\left({ }^{14} \mathrm{~N}\right)=\frac{\mathrm{e}^{2} \mathrm{Qq}}{4 \mathrm{~h}}(3+\eta) \\
v_{-}\left({ }^{14} \mathrm{~N}\right)=\frac{\mathrm{e}^{2} \mathrm{Qq}}{4 \mathrm{~h}}(3-\eta) \\
v_{0}\left({ }^{14} \mathrm{~N}\right)=v_{+}\left({ }^{14} \mathrm{~N}\right)-v_{-}\left({ }^{14} \mathrm{~N}\right)=\frac{\mathrm{e}^{2} \mathrm{Qq}}{2 \mathrm{~h}} \eta
\end{array}
$$

The natural abundance of the ${ }^{35} \mathrm{Cl}$ isotope is high (75.4\%), and the NQR frequencies are typically between 30 and $40 \mathrm{MHz}$, so it is possible to use the pure NQR experimental technique. The natural abundance of the ${ }^{14} \mathrm{~N}$ isotope is very high $(99.636 \%)$, but the NQR signals are weak, and the NQR frequencies are typically between 0.5 and $4 \mathrm{MHz}$, so the use of the ${ }^{1} \mathrm{H}_{-}{ }^{14} \mathrm{~N}$ NQDR technique is preferred to the use of pure NQR. The natural abundance of the ${ }^{17} \mathrm{O}$ isotope is rather low $(0.037 \%)$, and the NQR frequencies are typically below $5 \mathrm{MHz}$, so the use of several ${ }^{1} \mathrm{H}_{-}{ }^{17} \mathrm{O}$ NQDR techniques is required in this case.

\section{${ }^{1} \mathrm{H}-{ }^{14} \mathrm{~N}-\mathrm{NQDR}$}

Different double resonance techniques based on magnetic field cycling were used to detect ${ }^{14} \mathrm{~N}$ NQR frequencies. The proton spin system was polarized in $B_{0}=0.75 \mathrm{~T}$ for $30 \mathrm{~s}$. Then the sample was pneumatically transferred into another magnet within $0.1 \mathrm{~s}$, where it was left for $0.3 \mathrm{~s}$. In this other magnet, the magnetic field can be varied continuously between zero and $0.1 \mathrm{~T}$. After its stay in this other magnet, the sample was pneumatically transferred back into the first magnet within $0.1 \mathrm{~s}$, and the proton NMR signal was measured immediately after the sample had been stopped in the first magnet.

We used ${ }^{1} \mathrm{H}-{ }^{14} \mathrm{~N}$ cross-relaxation spectroscopy [27-29] as a first method. In this method, the sample is left to relax in a low magnetic field for a fixed time $\tau$ (in our case $\tau=$ $0.5 \mathrm{~s}$ ), and the low magnetic field is varied between the magnetic field cycles in steps of approximately $0.5 \mathrm{mT}$, corresponding to a step in the proton Larmor frequency $\nu_{\mathrm{L}}$ of $20 \mathrm{kHz}$. A proton Larmor frequency range of between 0 and $4 \mathrm{MHz}$ is usually scanned by this technique. When the proton Larmor frequency $\nu_{\mathrm{L}}$ matches a ${ }^{14} \mathrm{~N} \mathrm{NQR}$ frequency $\nu_{\mathrm{Q}}$, the proton spin-lattice relaxation time shortens, which results in a decrease in the proton NMR signal after the cycle. In some cases, especially at higher proton Larmor frequencies, a step of $40 \mathrm{kHz}$ can be used. On the other hand, around $\nu_{\mathrm{L}}=\nu_{\mathrm{Q}}$, the step is reduced to $10 \mathrm{kHz}$ to improve the resolution.

In a second step, we used the solid-effect technique [30]. In this method, the low magnetic field was fixed at a value $B$, corresponding to the proton Larmor frequency $v_{\mathrm{L}}=\gamma_{H} B / 2 \pi$, and the sample was irradiated in the low magnetic field with a strong rf magnetic field for $0.5 \mathrm{~s}$ at variable frequencies. When the frequency $\nu$ of the $\mathrm{rf}$ magnetic field is equal to $\nu=\nu_{\mathrm{Q}} \pm \nu_{\mathrm{L}}$, simultaneous spin flips take place in both the ${ }^{1} \mathrm{H}$ and the ${ }^{14} \mathrm{~N}$ spin systems. As a result, the proton magnetization decreases. The experiment is repeated at a few low magnetic field values to clarify the spectrum and get rid of signal artefacts caused by the direct proton absorption of the rf power at multiples of the proton Larmor frequency and the level crossing signals produced by the higher harmonics of the rf magnetic field.

We then used the two-frequency irradiation technique as a final technique combining the three ${ }^{14} \mathrm{~N}$ NQR frequencies from a given nitrogen site [31]. Here, the proton Larmor frequency $\nu_{\mathrm{L}}$ in the low magnetic field is set in resonance with the lowest ${ }^{14} \mathrm{~N} N Q R$ frequency $\nu_{0}$, and the sample is irradiated with two $\mathrm{rf}$ magnetic fields at the frequencies $\nu_{1}=\nu$ and $\nu_{2}=\nu+\nu_{0}$. When $\nu_{1}=\nu_{-}$and $\nu_{2}=\nu_{-}+v_{0}=v_{+}$, the proton relaxation rate in the low magnetic field increases and, as a result, the proton NMR signal at the end of the magnetic field cycle drops to a low value. This technique is applied when attempting to solve complex ${ }^{14} \mathrm{~N}$ NQR spectra in order to help distinguish between triplets corresponding to various nitrogen positions in the crystal.

\section{${ }^{1} \mathrm{H}-{ }^{17} \mathrm{O}$ NQDR}

The ${ }^{17} \mathrm{O}$ NQR frequencies in 5-chloroquinol were first measured by the Slusher and Hahn technique [32]. The sample was pneumatically moved between two magnets with magnetic fields of $B_{0}=0.75 \mathrm{~T}$ and zero. The proton spin system was polarized in the high magnetic field $B_{0}$ for $30 \mathrm{~s}$ and then transferred to the zero magnetic field for $0.8 \mathrm{~s}$. Then the sample was transferred back into the first magnet and the intensity of the proton NMR signal was measured immediately after the sample stopped in the high magnetic field. In the static zero magnetic field, the sample was irradiated with a phase-modulated $\mathrm{rf}$ magnetic field of frequency $\nu$ and amplitude $\sim 3 \mathrm{mT}$. Square-wave $180^{\circ}$ phase 
modulation was used with a frequency of $1.7 \mathrm{kHz}$. The frequency $\nu$ was changed between the repetitive magnetic field cycles in steps of $20 \mathrm{kHz}$. The frequency range between 1.0 and $5.0 \mathrm{MHz}$ was scanned by the frequency $\nu$. Two dips corresponding to the ${ }^{17} \mathrm{O}$ NQR frequencies $\nu_{3 / 2-1 / 2}$ and $\nu_{5 / 2-3 / 2}$ are usually observed by this technique. The third dip at the highest ${ }^{17} \mathrm{O}$ NQR frequency $\nu=\nu_{5 / 2-1 / 2}$ has (as shown in [33]) a much lower intensity than the other two dips, and is usually not observed by the Slusher and Hahn technique.

During the second part of the experiment we used the two-frequency irradiation technique [34] to determine the dipolar structure of the ${ }^{1} \mathrm{H}^{17} \mathrm{O}$ NQDR lines. Two $\mathrm{rf}$ magnetic fields of frequencies $\nu_{1}$ and $\nu_{2}$ were applied in repetitive pulses at frequencies $\nu_{1}, \nu_{2}, \nu_{1}, \nu_{2} \ldots$ The duration of a pulse was $1 \mathrm{~ms}$. The amplitude of the rf magnetic field during the measurement of the dipolar structure of the NQDR lines at $\nu=\nu_{3 / 2-1 / 2}$ and $\nu=\nu_{5 / 2-3 / 2}$ was reduced to $0.3 \mathrm{mT}$. The dipolar structure of the NQDR line with the highest frequency was measured with an rf magnetic field of amplitude $3 \mathrm{mT}$, due to the lower transition probability per unit time.

The dipolar structure of a NQDR line was determined in two experiments. The first of the two frequencies, say $\nu_{1}$, was fixed at the lower edge of the NQDR line, and the line was scanned at the second frequency $\nu_{2}$ in steps of $5 \mathrm{kHz}$. No drop in the proton NMR signal was observed when $\nu_{1}=$ $\nu_{2}$. The strongest drop in the proton NMR signal was observed when $\nu_{2}$ was in the upper part of the NQDR line. To determine the dipolar structure of the lower part of the NQDR line, the experiment was repeated with the frequency $\nu_{1}$ fixed at the upper edge of the NQDR line. The dipolar structures of the three NQDR lines determined by twofrequency irradiation were analyzed according to [35].

\section{${ }^{35} \mathrm{Cl}-\mathrm{NQR}$}

The ${ }^{35} \mathrm{Cl}-\mathrm{NQR}$ spectra of clioquinol and cloxiquine were taken at $77 \mathrm{~K}$. The NQR signals assigned to $\mathrm{Cl}$ nuclei were weak ( $\mathrm{S} / \mathrm{N}=3$ after 1000 accumulations), and the resonance line was wide [full width at half maximum (FWHM) was $27 \mathrm{kHz}$, so the classical Hahn sequence $\pi-\tau-2 \pi$ was applied. In the NQR of a powdered sample, both excitation and signal reception depend on the relative orientations of the crystallites with respect to the coil axis, so the pulse sequence was optimized; the optimized pulse length was 5 $\mu$ s and the interval between the pulses was $90 \mu$ s. The NQR lineshape was obtained from the fast Fourier transform (FFT) of both half-echo signals after 1000 accumulations for the desired signal-to-noise ratio. The repetition time of the scans was $200 \mathrm{~ms}$. The accuracy of the ${ }^{35} \mathrm{Cl}-\mathrm{NQR}$ frequency determination was $\sim 10 \mathrm{kHz}$.

\section{DFT calculations}

Quantum chemical calculations were carried out within the GAUSSIAN03 ${ }^{\mathrm{TM}}$ code [36], which was run on the CRAY supercomputer at the Poznan Supercomputer and Network Centre (PCSS) in Poznan, Poland. All calculations were performed within the density functional theory (DFT) with a hybrid exchange-correlation functional, B3LYP (the three-parameter exchange functional of Becke B3 [37] combined with the Lee-Yang-Parr correlation functional LYP [38]), using the extended basis set $6-311++\mathrm{G}^{* *}$ with polarization and diffuse functions. The calculations were carried out under the assumption of the crystallographic as well as the partially optimized geometry, where only the positions of the hydrogen atoms were allowed to relax during optimization (as performed using the Berny algorithm), while those of all other atoms remained frozen. The NQR parameters-quadrupole coupling constants, asymmetry parameters and frequencies at all nitrogen atomswere calculated assuming different polymorphic forms, which differed in the molecular aggregations formed as a result of intermolecular interactions.

Theoretical analysis of the intermolecular interactions was performed according to the quantum theory of atoms in molecules theory (QTAIM) [39], and the topological parameters were calculated, including the bond critical points (BCPs), ring critical points (RCPs), Laplacian of the electron density $(\Delta \rho)$, ellipiticity of the bond $(\varepsilon)$, the total electron energy density at the $\mathrm{BCP}\left(H_{\mathrm{BCP}}\right)$ and its components: the local kinetic energy density $\left(G_{\mathrm{BCP}}\right)$ and the local potential energy density $\left(V_{\mathrm{BCP}}\right)$. Because of the dependence of the basis on atomic position (i.e., the basis set superposition error, BSSE), small interaction energies are often overestimated, so the interaction energies were corrected for BSSE by the standard counterpoise (CP) method [40]. Another correction was made for the zero point vibrational energies (ZPVEs). However, this approach is not useful for intramolecular or multiple hydrogen bonds, so the energy of the interactions was calculated according to Espinosa [41].

\section{Results and discussion}

The ${ }^{35} \mathrm{Cl}-\mathrm{NQR}$ spectra of clioquinol and cloxiquine are presented in Fig. 2. Only one resonance line is observed in each, at $35.170 \mathrm{MHz}$ for clioquinol and at $34.787 \mathrm{MHz}$ for cloxiquine at $77 \mathrm{~K}$. All of the molecules are thus crystallographically equivalent in each of these compounds, which is in agreement with the X-ray data [42-45].

The ${ }^{1} \mathrm{H}^{14} \mathrm{~N}$ NQDR spectra of clioquinol and cloxiquine as obtained by the solid-effect technique at $T=295 \mathrm{~K}$ are presented in Fig. 3. Seven NQDR lines are unambiguously 

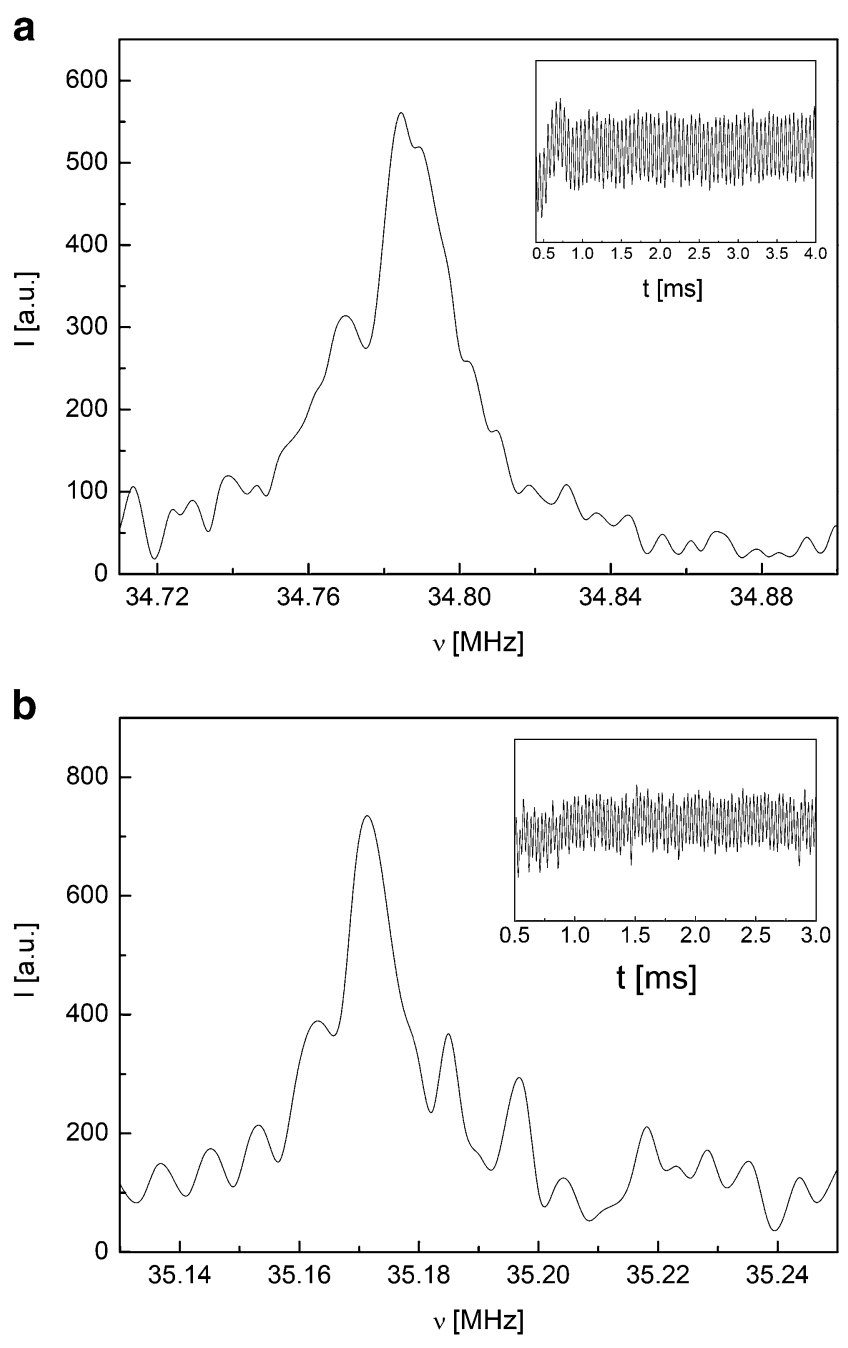

Fig. 2 The ${ }^{35} \mathrm{Cl}-\mathrm{NQR}$ spectra of clioquinol and cloxiquine at $T=77 \mathrm{~K}$

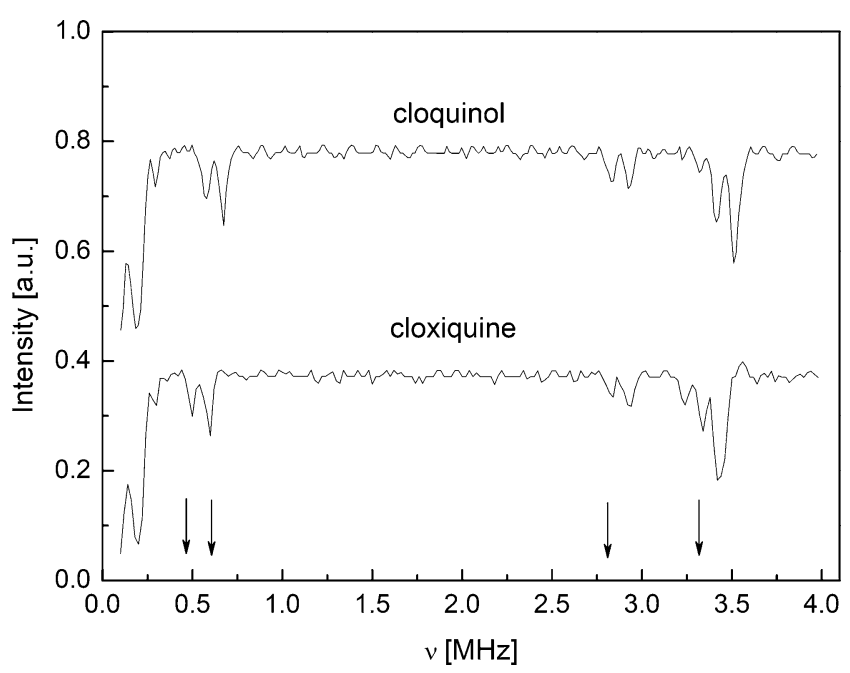

Fig. 3 The ${ }^{1} \mathrm{H}^{14}{ }^{\mathrm{N}}$ solid-effect double-resonance spectra of clioquinol and cloxiquine at $T=295 \mathrm{~K}$. The proton Larmor frequency is $\nu_{\mathrm{L}}=$ $100 \mathrm{kHz}$ resolved in each spectrum. However, as shown in [29], the intensities of the NQDR lines differ strongly when the population of the energy levels of the nitrogen spin system approaches the Boltzmann population more slowly than that of the energy levels of the proton spin system. A triplet is usually observed at $\nu=\nu_{+}-\nu_{\mathrm{L}}, \nu_{+}$and $\nu_{+}+\nu_{\mathrm{L}}$. The solideffect lines $\nu=\nu_{0}-\nu_{\mathrm{L}}$ and $\nu=\nu_{-}-\nu_{\mathrm{L}}$ are usually missing. With this in mind, and utilizing the previously recorded cross-relaxation spectra, we can determine the ${ }^{14} \mathrm{~N}$ NQR frequencies as $3.330 \mathrm{MHz}, 2.830 \mathrm{MHz}$ and $0.500 \mathrm{MHz}$ for cloxiquine and $3.410 \mathrm{MHz}, 2.830 \mathrm{MHz}$ and $0.580 \mathrm{MHz}$ for clioquinol. The accuracy is within $\pm 10-20 \mathrm{kHz}$. A more precise determination of the ${ }^{14} \mathrm{~N}$ NQR frequencies to an accuracy of $\pm 2 \mathrm{kHz}$ was obtained using the two-frequency irradiation technique. The ${ }^{14} \mathrm{~N}$ NQR frequencies of $3.328 \mathrm{MHz}, 2.828 \mathrm{MHz}$ and $0.500 \mathrm{MHz}$ for cloxiquine and $3.407 \mathrm{MHz}, 2.832 \mathrm{MHz}$ and $0.575 \mathrm{MHz}$ for clioquinol are not far from the frequencies determined from the solid-
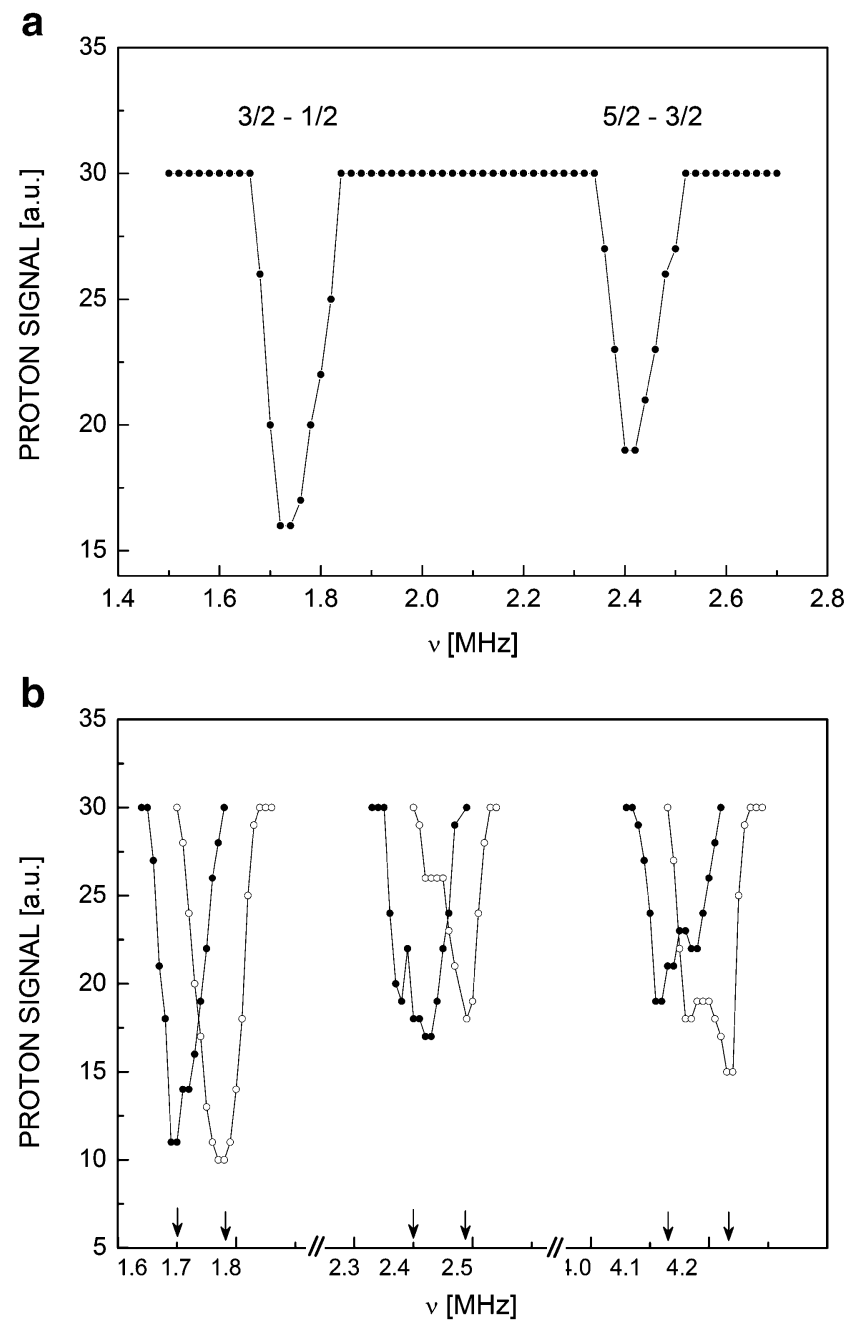

Fig. 4a-b The ${ }^{1} \mathrm{H}-{ }^{17} \mathrm{O}$ double resonance spectra of cloxiquine at $T=$ $213 \mathrm{~K}$, as measured by the Slusher and Hahn technique (a) and by the two-frequency irradiation technique (b) 
effect spectra. All nitrogen positions are crystallographically equivalent in cloxiquine as well as in clioquinol.

The ${ }^{1} \mathrm{H}^{17} \mathrm{O}$ NQDR spectrum was only measured for cloxiquine. Due to the low natural abundance of ${ }^{17} \mathrm{O}$ $(0.037 \%)$, the sensitivity of the NQDR technique strongly depends on the proton spin-lattice relaxation time in zero magnetic field, which should be about $1 \mathrm{~s}$ or more to observe the NQDR dips. For cloxiquine we obtained a sufficiently long proton spin-lattice relaxation time in zero magnetic field by reducing the temperature of the sample to $213 \mathrm{~K}$. For clioquinol we also varied the temperature of the sample, but within the working range of the spectrometer $(130-400 \mathrm{~K})$ we did not observe a sufficiently long proton $T_{1}$ in zero magnetic field. This is presumably the effect of the contribution of ${ }^{127}$ I to the spin-lattice relaxation of the proton dipolar system.

The ${ }^{1} \mathrm{H}^{-17} \mathrm{O}$ NQDR spectrum of cloxiquine as obtained by the Slusher and Hahn technique is presented in Fig. 4a. Two NQDR dips are observed at frequencies of 2.425 and $1.750 \mathrm{MHz}$. These two dips correspond to the ${ }^{17} \mathrm{O} \mathrm{NQR}$ frequencies $\nu_{5 / 2-3 / 2}$ and $\nu_{3 / 2-1 / 2}$, respectively. The third dip expected at the frequency $v_{5 / 2-1 / 2}=v_{5 / 2-3 / 2}+v_{3 / 2-1 / 2}=$ $4.175 \mathrm{MHz}$ is too weak to be observed by the Slusher and Hahn technique.

The dipolar structure of the three ${ }^{17} \mathrm{O}$ NQR lines, as measured by the two-frequency irradiation technique, is presented in Fig. 4b. The 5/2-1/2 transition is also observed by this technique. The dipolar structure of the three ${ }^{17} \mathrm{O}$ NQR lines is not well resolved due to the dipolar protonproton interaction. Nevertheless, it is still possible to determine the proton-oxygen distance plus the polar angle $\theta$ and the azimuthal angle $\phi$ describing the orientation of the $\mathrm{O}-\mathrm{H}$ bond in the principal-axis frame of the EFG tensor

Table 1 The experimental NQR parameters for clioquinol and cloxiquine $\left({ }^{35} \mathrm{Cl} \mathrm{NQR}\right.$ frequency, ${ }^{14} \mathrm{~N} \mathrm{NQR}$ frequencies $\nu_{+}, \nu_{-}$and $\nu_{0},{ }^{17} \mathrm{O}$ NQR frequencies $\nu_{3 / 2-1 / 2}, \nu_{3 / 2-1 / 2}$ and $\nu_{3 / 2-1 / 2}$, the widths $\delta \nu_{3 / 2}$ $1 / 2, \delta \nu_{3 / 2-1 / 2}$ and $\delta \nu_{3 / 2-1 / 2}$ of the NQDR lines, the quadrupole coupling constants $\mathrm{e}^{2} \mathrm{qQ} / \mathrm{h}$, and the asymmetry parameters $\eta$ of the EFG tensor, at the position of the oxygen nucleus from the widths of the dipolar split ${ }^{17} \mathrm{O}$ NQR lines [34]. The results are as follows: the $\mathrm{O}-\mathrm{H}$ distance is $0.99 \pm 0.01 \AA$; the $\mathrm{O}-\mathrm{H}$ bond lies in the $x z$ plane of the EFG tensor $\left(\varphi=0^{\circ}\right)$ and makes an angle $\theta=50^{\circ} 5^{\circ}$ with the principal axis $z$ of the EFG tensor.

The experimental values of the ${ }^{14} \mathrm{~N},{ }^{17} \mathrm{O}$ and ${ }^{35} \mathrm{Cl} \mathrm{NQR}$ frequencies, together with the values of $\mathrm{e}^{2} \mathrm{Qqh}^{-1}$ and $\eta$ calculated using these frequencies, are collected in Table 1 for both compounds. As follows from these results, all of the clioquinol as well as the cloxiquine molecules in the elementary cell are equivalent within the resolution of the experiment, which is in good agreement with the X-ray data, according to which clioquinol crystallizes in the monoclinic system $\mathrm{P} 2 / \mathrm{c}$ or P2/a $[42,43]$ while cloxiquine crystallizes in the orthorhombic system Fdd2 (form I) or the monoclinic system P2/c (form II) [44, 45].

Parameters of the elementary cells of form 1 of cloxiquine at $90 \mathrm{~K}$ [45] and at RT [44], as determined by crystallographic methods, differ insignificantly (by $92.5 \AA^{3}$ in volume; $V(90 \mathrm{~K})=3032.5$ and $\left.V(\mathrm{RT})=3126 \AA^{3}\right)$, which suggests that a phase transition does not take place over this range of temperatures. Because there is only one type of each site (nitrogen, oxygen, chlorine or iodine) in clioquinol and cloxiquine, assigning the NQR frequencies to particular sites is a straightforward task. The differences in the reproduced NQR parameters for forms I and II of cloxiquine (see Table 2) are large enough to allow the unambiguous identification of the present polymorphic form as form I. This conclusion is based on two factors: significant differences between $\mathrm{e}^{2} \mathrm{Qq} / \mathrm{h}$ and $\eta$, especially at the ${ }^{17} \mathrm{O}$ site for both forms (see Table 2), and, more importantly, the much better correlation between the experimental and DFT-calculated ${ }^{14} \mathrm{~N}$ and ${ }^{17} \mathrm{O}$ NQR

the proton-oxygen distance $\mathrm{R}(\mathrm{O}-\mathrm{H})$, the angle $\theta$ between the $\mathrm{O}-\mathrm{H}$ bond and the principal axis $z$ of the EFG tensor, and the angle $\phi$ between the projection of the $\mathrm{O}-\mathrm{H}$ bond on the $x y$ plane and the principal axis $x$ of the EFG tensor)

\begin{tabular}{|c|c|c|c|c|c|c|c|c|}
\hline Compound & Site & $\nu$ in $\mathrm{MHz}(\delta \nu$ in $\mathrm{kHz})$ & $e^{2} Q q h^{-1}(\mathrm{MHz})$ & $\eta$ & $R(\mathrm{O}-\mathrm{H})(\AA)$ & $\theta$ & $\phi$ & $T(\mathrm{~K})$ \\
\hline \multirow[t]{5}{*}{ Cloxiquine } & \multirow[t]{2}{*}{$\mathrm{N}$} & $\begin{array}{l}3.328 \\
2.828\end{array}$ & \multirow[t]{2}{*}{4.104} & \multirow[t]{2}{*}{0.244} & \multirow[t]{2}{*}{-} & \multirow[t]{2}{*}{-} & \multirow[t]{2}{*}{-} & \multirow[t]{2}{*}{295} \\
\hline & & 0.500 & & & & & & \\
\hline & \multirow[t]{2}{*}{$\mathrm{O}$} & $\begin{array}{l}4.175(120) \\
2.425(120)\end{array}$ & \multirow[t]{2}{*}{8.610} & \multirow[t]{2}{*}{0.622} & \multirow[t]{2}{*}{0.99} & \multirow[t]{2}{*}{$50^{\circ}$} & \multirow[t]{2}{*}{$0^{\circ}$} & \multirow[t]{2}{*}{213} \\
\hline & & $1.750(110)$ & & & & & & \\
\hline & $\mathrm{Cl}$ & $34.787(21)$ & $69.574^{\mathrm{a}}$ & 0 & - & - & - & 77 \\
\hline \multirow[t]{3}{*}{ Clioquinol } & \multirow[t]{2}{*}{$\mathrm{N}$} & $\begin{array}{l}3.407 \\
2.831\end{array}$ & \multirow[t]{2}{*}{4.159} & \multirow[t]{2}{*}{0.276} & \multirow[t]{2}{*}{-} & \multirow[t]{2}{*}{-} & \multirow[t]{2}{*}{-} & \multirow[t]{2}{*}{295} \\
\hline & & 0.575 & & & & & & \\
\hline & $\mathrm{Cl}$ & $35.170(22)$ & $71.420^{\mathrm{a}}$ & 0 & - & - & - & 77 \\
\hline
\end{tabular}

${ }^{\text {a }}$ Calculated under the assumption $\eta=0$ 
Table 2 The NQR parameters calculated at the B3LYP/6-311++G(d,p) level of theory for different polymorphic forms of clioquinol and cloxiquine

\begin{tabular}{|c|c|c|c|c|c|c|c|c|c|c|c|}
\hline \multirow[t]{2}{*}{ Compound } & \multirow[t]{2}{*}{ Form } & \multirow[t]{2}{*}{ Site } & \multicolumn{3}{|l|}{ Monomer } & \multicolumn{3}{|l|}{ Dimer } & \multicolumn{3}{|l|}{ Stacked dimers } \\
\hline & & & $\begin{array}{l}\nu_{+}, \nu_{-}, \nu_{0} \\
\nu_{5 / 2-1 / 2,}, \nu_{5 / 2-3 / 2}, \nu_{3 / 2-1 / 2} \\
\nu(\mathrm{MHz})\end{array}$ & $\begin{array}{l}e^{2} Q q h^{-1} \\
(\mathrm{MHz})\end{array}$ & $\eta$ & $\begin{array}{l}\nu_{+}, \nu_{-}, \nu_{0} \\
\nu_{5 / 2-1 / 2,}, \nu_{5 / 2-3 / 2}, \nu_{3 / 2-1 / 2} \\
\nu(\mathrm{MHz})\end{array}$ & $\begin{array}{l}e^{2} Q q h^{-1} \\
(\mathrm{MHz})\end{array}$ & $\eta$ & $\begin{array}{l}\nu_{+}, \nu_{-}, \nu_{0} \\
\nu_{5 / 2-1 / 2,}, \nu_{5 / 2-3 / 2}, \nu_{3 / 2-1 / 2} \\
\nu(\mathrm{MHz})\end{array}$ & $\begin{array}{l}e^{2} Q q h^{-1} \\
(\mathrm{MHz})\end{array}$ & $\eta$ \\
\hline \multirow[t]{15}{*}{ Cloxiquine } & \multirow[t]{5}{*}{ Form I RT [44] $\mathrm{opt}^{\mathrm{a}}$} & \multirow[t]{2}{*}{$\mathrm{N}$} & $\begin{array}{l}3.969 \\
3.285\end{array}$ & \multirow[t]{2}{*}{4.836} & \multirow[t]{2}{*}{0.283} & $\begin{array}{l}3.607 \\
3.079\end{array}$ & \multirow[t]{2}{*}{4.457} & \multirow[t]{2}{*}{0.237} & $\begin{array}{l}3.610 \\
3.080\end{array}$ & \multirow[t]{2}{*}{4.460} & \multirow[t]{2}{*}{0.238} \\
\hline & & & 0.684 & & & 0.528 & & & 0.530 & & \\
\hline & & \multirow[t]{2}{*}{$\mathrm{O}$} & $\begin{array}{l}5.116 \\
2.907\end{array}$ & \multirow[t]{2}{*}{10.424} & \multirow[t]{2}{*}{0.680} & $\begin{array}{l}4.717 \\
2.764\end{array}$ & \multirow[t]{2}{*}{9.776} & \multirow[t]{2}{*}{0.599} & $\begin{array}{l}4.732 \\
2.757\end{array}$ & \multirow[t]{2}{*}{9.766} & \multirow[t]{2}{*}{0.614} \\
\hline & & & 2.208 & & & 1.953 & & & 1.974 & & \\
\hline & & $\mathrm{Cl}$ & 33.634 & 67.074 & 0.093 & 33.663 & 67.145 & 0.090 & 33.881 & 67.611 & 0.082 \\
\hline & \multirow[t]{5}{*}{ Form I 90 K [45] opt $^{\mathrm{a}}$} & \multirow[t]{2}{*}{$\mathrm{N}$} & $\begin{array}{l}4.007 \\
3.310\end{array}$ & \multirow[t]{2}{*}{4.878} & \multirow[t]{2}{*}{0.286} & $\begin{array}{l}3.860 \\
3.251\end{array}$ & \multirow[t]{2}{*}{4.741} & \multirow[t]{2}{*}{0.257} & $\begin{array}{l}3.881 \\
3.256\end{array}$ & 4.758 & 0.263 \\
\hline & & & 0.698 & & & 0.609 & & & 0.626 & & \\
\hline & & $\mathrm{O}$ & $\begin{array}{l}4.838 \\
2.730\end{array}$ & 9.817 & 0.699 & $\begin{array}{l}4.621 \\
2.644\end{array}$ & 9.453 & 0.662 & $\begin{array}{l}4.635 \\
2.639\end{array}$ & 9.452 & 0.676 \\
\hline & & & 2.108 & & & 1.977 & & & 1.996 & & \\
\hline & & $\mathrm{Cl}$ & 34.005 & 67.859 & 0.082 & 33.987 & 67.826 & 0.081 & 34.224 & 68.330 & 0.072 \\
\hline & Form II $90 \mathrm{~K}$ [45] opt $\mathrm{t}^{\mathrm{a}}$ & $\mathrm{N}$ & $\begin{array}{l}3.884 \\
3.202\end{array}$ & 4.724 & 0.289 & $\begin{array}{l}3.666 \\
3.084\end{array}$ & 4.500 & 0.259 & $\begin{array}{l}3.706 \\
3.100\end{array}$ & 4.537 & 0.267 \\
\hline & & & 0.683 & & & 0.583 & & & 0.606 & & \\
\hline & & $\mathrm{O}$ & $\begin{array}{l}4.802 \\
2.547\end{array}$ & 9.402 & 0.855 & $\begin{array}{l}4.568 \\
2.437\end{array}$ & 8.975 & 0.840 & $\begin{array}{l}4.528 \\
2.405\end{array}$ & 8.981 & 0.834 \\
\hline & & & 2.256 & & & 2.131 & & & 2.123 & & \\
\hline & & $\mathrm{Cl}$ & 34.038 & 67.928 & 0.081 & 34.030 & 67.918 & 0.079 & 34.195 & 68.273 & 0.072 \\
\hline Clioquinol & Form I [43] opt $^{\mathrm{a}}$ & $\mathrm{N}$ & $\begin{array}{l}4.06 \\
3.41\end{array}$ & 4.979 & 0.259 & $\begin{array}{l}3.71 \\
3.22\end{array}$ & 4.621 & 0.210 & $\begin{array}{l}3.69 \\
3.21\end{array}$ & 4.602 & 0.206 \\
\hline & & & 0.64 & & & 0.49 & & & 0.47 & & \\
\hline & & $\mathrm{O}$ & $\begin{array}{l}5.189 \\
2.866\end{array}$ & 10.403 & 0.754 & $\begin{array}{l}4.830 \\
2.781\end{array}$ & 9.915 & 0.646 & $\begin{array}{l}4.836 \\
2.765\end{array}$ & 9.886 & 0.665 \\
\hline & & & 2.424 & & & 2.049 & & & 2.072 & & \\
\hline & & $\mathrm{Cl}$ & 34.372 & 68.566 & 0.088 & 34.413 & 68.657 & 0.086 & 34.589 & 69.012 & 0.085 \\
\hline & & I & $\begin{array}{l}889.136 \\
585.596\end{array}$ & 1971.039 & 0.104 & $\begin{array}{l}886.267 \\
587.800\end{array}$ & 1964.244 & 0.109 & $\begin{array}{l}894.756 \\
594.783\end{array}$ & 1985.258 & 0.082 \\
\hline & & & 303.540 & & & 298.467 & & & 299.972 & & \\
\hline & Form I [42]opt $\mathrm{t}^{\mathrm{a}}$ & $\mathrm{N}$ & $\begin{array}{l}4.06 \\
3.53\end{array}$ & 5.062 & 0.207 & $\begin{array}{l}3.70 \\
3.34\end{array}$ & 4.692 & 0.154 & $\begin{array}{l}3.69 \\
3.33\end{array}$ & 4.680 & 0.152 \\
\hline & & & 0.52 & & & 0.36 & & & 0.36 & & \\
\hline & & $\mathrm{O}$ & $\begin{array}{l}5.033 \\
2.834\end{array}$ & 10.202 & 0.704 & $\begin{array}{l}4.674 \\
2.732\end{array}$ & 9.673 & 0.605 & $\begin{array}{l}4.686 \\
2.716\end{array}$ & 9.654 & 0.628 \\
\hline & & & 2.199 & & & 1.941 & & & 1.969 & & \\
\hline & & $\mathrm{Cl}$ & 35.335 & 70.551 & 0.071 & 35.382 & 70.655 & 0.068 & 35.539 & 70.975 & 0.066 \\
\hline & & I & $\begin{array}{l}897.710 \\
596.647\end{array}$ & 1991.812 & 0.084 & $\begin{array}{l}895.961 \\
597.043\end{array}$ & 1987.492 & 0.089 & $\begin{array}{l}903.91 \\
601.23\end{array}$ & 2007.117 & 0.060 \\
\hline & & & 301.062 & & & 300.708 & & & 302.673 & & \\
\hline
\end{tabular}

${ }^{\text {a }}$ Partially optimized geometry

frequencies (for form I, the correlation coefficient and curve fit standard errors are 0.997 and $0.025 \mathrm{MHz}$ at RT, and 0.9956 and $0.036 \mathrm{MHz}$ at $90 \mathrm{~K}$, but for form II they are only 0.986 and $0.105 \mathrm{MHz}$ ); see Fig. 5a. It is worth noting that the NQR frequency at the ${ }^{35} \mathrm{Cl}$ site is less useful. Although it is more sensitive to the influence of temperature than those at the ${ }^{17} \mathrm{O}$ and ${ }^{14} \mathrm{~N}$ sites, the ${ }^{35} \mathrm{Cl}$ site is distinct and thus less sensitive to structural changes in the stacked dimers.

Surprisingly, the NQR parameters for clioquinol are better reproduced when assuming the X-ray structure from [43], denoted form Ia, rather than that from [42], denoted form $\mathrm{Ib}$ (see Tables 3 and 4), which suggests that the first structure is better resolved. However, the residual factor 


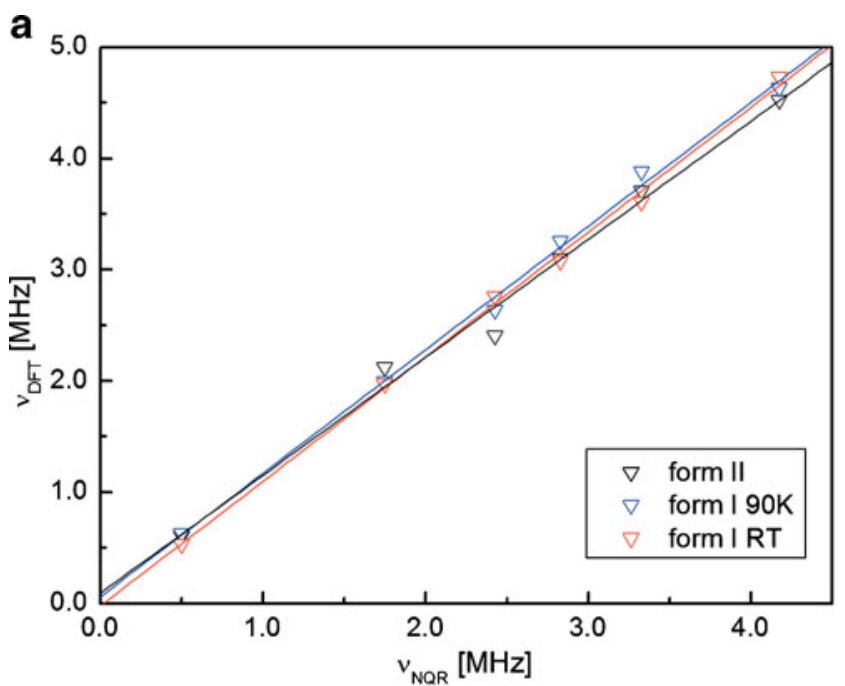

b

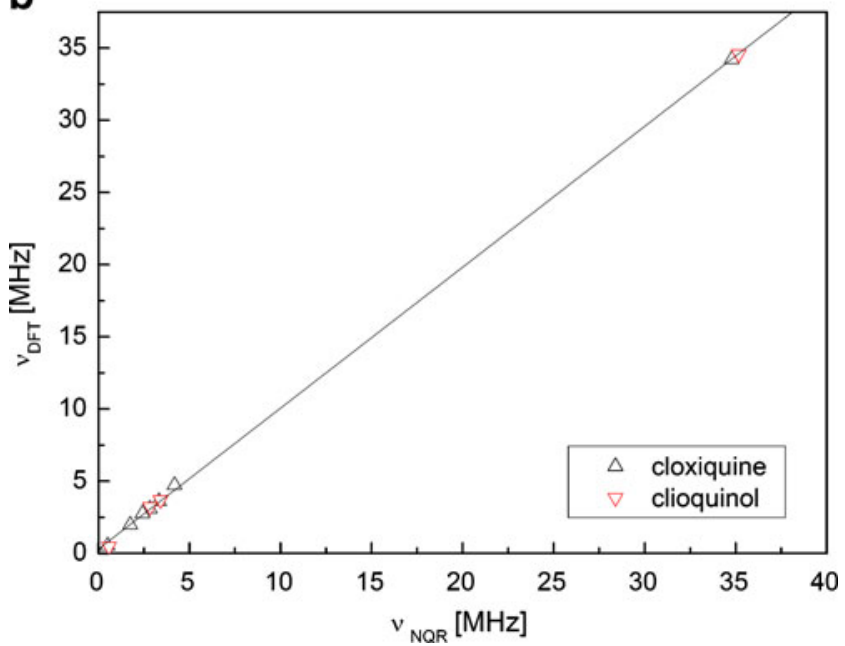

Fig. 5a-b The correlation between the experimental and calculated NQR frequencies. a Cloxiquine (form I at RT and $90 \mathrm{~K}$, form II). b Cloxiquine (form I), clioquinol (form Ia)

given by the authors for this structure is only $10 \%$, whereas it is $8.4 \%$ for the second structure. The NQR parameters $\mathrm{e}^{2} \mathrm{Qqh}^{-1}$ and $\eta$ and the frequencies at all quadrupolar nuclei were calculated at the B3LYP/6-311++G(d,p) level using $\mathrm{X}$-ray data for clioquinol and cloxiquine (for both polymorphic forms) and assuming different molecular aggregations (monomer, dimer, stacked dimers) are formed by the intermolecular interactions. The results are collected in Table 2. The orientations of the principal axes of the EFG tensors at each site are shown in Fig. 6a-c. Good reproduction accuracy of $\mathrm{e}^{2} \mathrm{Qqh}^{-1}$ and $\eta$ and the NQR frequencies at all quadrupolar nuclei $\left({ }^{14} \mathrm{~N},{ }^{17} \mathrm{O}\right.$ and $\left.{ }^{35} \mathrm{Cl}\right)$ at the chosen DFT level (correlation coefficients as high as 0.999 , and curve fit standard errors as low as $0.38 \mathrm{MHz}$ ) requires the assumption of the optimized proton positions and consideration of the intermolecular bonding (see Tables 2, 3 and 4, and Fig. 5b), which is in a good agreement with the results of our previous studies of purines [23, 24] or polyhalogenobenzimidazoles [25]. Besides $\mathrm{e}^{2} \mathrm{Qqh}^{-1}$ and $\eta$, another criterion for checking the quality of reproduction of the EFG tensor by DFT is to compare the orientations of its principal axes as deduced from DFT as well as the $\mathrm{O}-\mathrm{H}$ bond length with those obtained from the experimental ${ }^{17} \mathrm{O}$ spectrum. The orientation of the $z$-axis of the EFG tensor determined from ${ }^{17} \mathrm{O}$ for cloxiquine is in good agreement with the results of the DFT calculations. DFT suggests that the $\mathrm{O}-\mathrm{H}$ bond, which lies in the $x z$ plane of the EFG tensor according to the NQDR results, deviates from the $x z$ plane by only $3^{\circ}$, and makes an angle of $44 \pm 1^{\circ}$ with the $z$-axis of the EFG tensor, while the experiment suggests $\theta=50 \pm 5^{\circ}$. The source of this slight discrepancy could be the neglect of other interactions aside from the hydrogen bonding. The $\mathrm{O}-\mathrm{H}$ bond length, $R(\mathrm{O}-\mathrm{H})$, as determined by ${ }^{17} \mathrm{O}$ NQDR for cloxiquine, is $R(\mathrm{O}-\mathrm{H})=(0.99 \pm 0.02) \AA$ (i.e., in good agreement with the X-ray data at $90 \mathrm{~K}$ [45] and the DFT partial optimization result for the monomer, $0.987 \AA$, and dimer, $1.011 \AA$ ). The $\mathrm{O}-\mathrm{H}$ bond length for clioquinol obtained from partial DFT optimization, $R(\mathrm{O}-\mathrm{H})=0.992 \AA$, is also much higher than those given in [42], i.e., $0.728 \AA$. The change in the $\angle \mathrm{OHN}$ angle describing the linearity of the $\mathrm{O}-\mathrm{H} \cdots \mathrm{N}$ bond influences the NQR parameters considerably, even more than the changes in its length, which explains why the NQR parameters for the structure with the optimized proton positions is reproduced more accurately.

Structural pattern

\section{Supramolecular synthon}

According to the crystallographic data, the hydrogen bonding patterns in solid clioquinol and cloxiquine (form I) [42-45] are isostructural to those in the parent 8quiniolinol [46]. The hydroxyl hydrogens are capable of forming multicenter bonds, i.e., bifurcated $\mathrm{O}-\mathrm{H} \cdots \mathrm{N}$ hydrogen bonds, one intramolecular and the other intermolecular, which simultaneously lead to the formation of fivemembered hydrogen-bonded chelate rings $[\mathrm{N}, \mathrm{C}(9), \mathrm{C}(8)$, $\mathrm{O}, \mathrm{H}(8)]$ and to the dimerization of the molecules, respectively, as indicated in Fig. 7a-c. Such patterns, usually termed supramolecular synthons [47], are, according to the X-ray data, independent of the polymorphic form. However, in the dimeric structures of form I of clioquinol and forms Ia and $\mathrm{Ib}$ of cloxiquine, the paired molecules in the units are twisted, while in cloxiquine (form II) they are not. Due to this subtle difference in the planarity of the dimeric structures which consist of the paired molecules linked by bifurcated hydrogen bonds in the units (twisted in form I, and planar in form II) [44, 45], accompanied by a change in the hydrogen bond lengths, the structural units in 


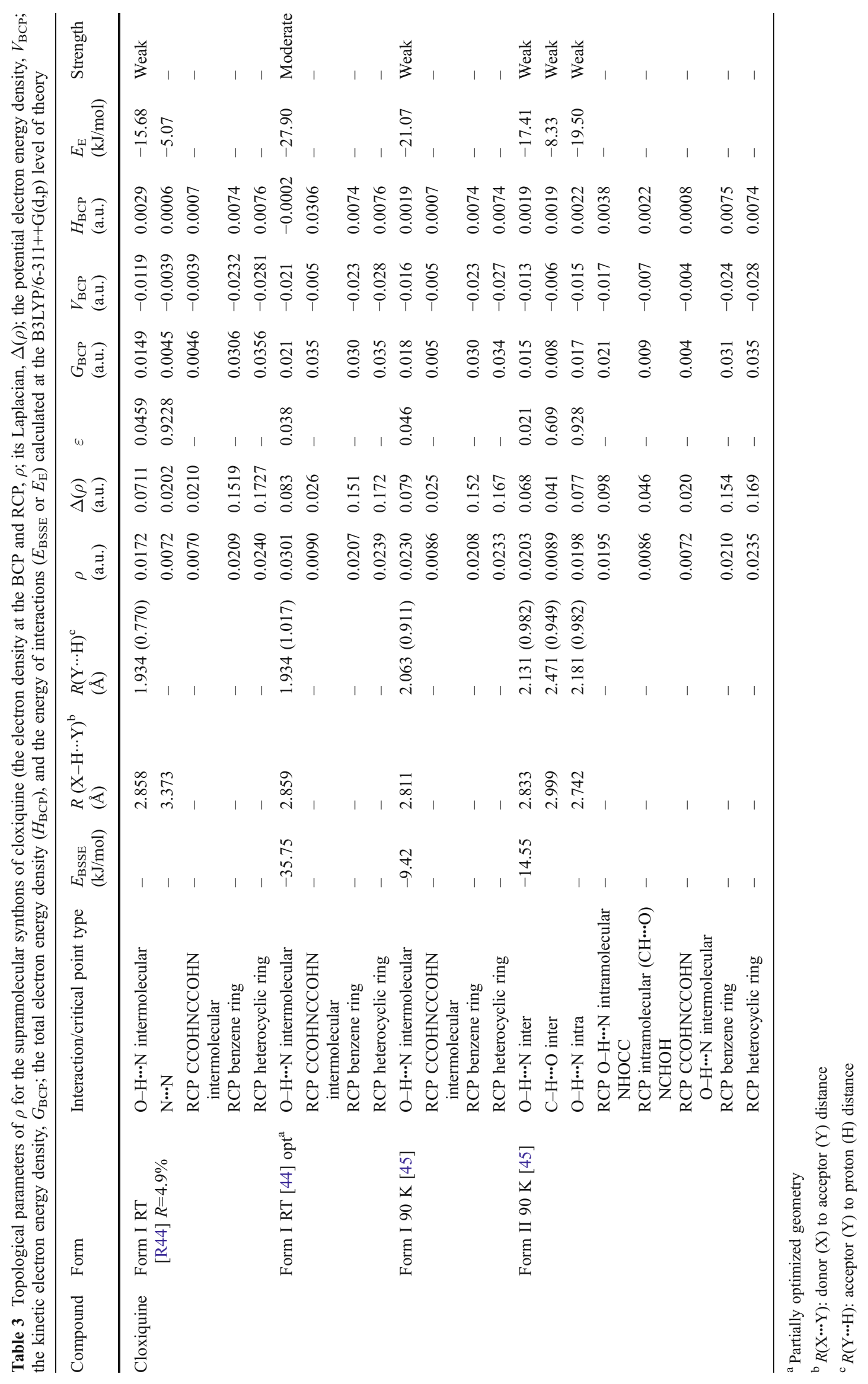


cloxiquine can be differently packed to yield two polymorphs. As follows from X-ray data, the intramolecular hydrogen bonds $\mathrm{O}-\mathrm{H} \cdots \mathrm{N}$ in clioquinol $\left(R_{\mathrm{O}-\mathrm{H} \cdots \mathrm{N}}=2.753\right.$ [42] or $2.818 \AA$ [43]) are longer than those reported in cloxiquine $\left(R_{\mathrm{O}-\mathrm{H} \cdots \mathrm{N}}=2.811\right.$ [44] and $2.747 \AA$ [45]), both of which are close to those in 8-quinolinol $\left(R_{\mathrm{O}-\mathrm{H} \cdots \mathrm{N}}=\right.$ $2.753 \AA[46])$. The intermolecular hydrogen bonds $\mathrm{O}-\mathrm{H} \cdots \mathrm{N}$ in form Ia of clioquinol $\left(R_{\mathrm{O}-\mathrm{H} \cdots \mathrm{N}}=2.792\right)$ are shorter than those reported in cloxiquine $\left(R_{\mathrm{O}-\mathrm{H} \cdots \mathrm{N}}=2.859\right.$ and $\left.2.833 \AA\right)$, but similar to those in 8-quinolinol $\left(R_{\mathrm{O}-\mathrm{H} \cdots \mathrm{N}}=2.793 \AA\right)$, while in form $\mathrm{Ib}$ of clioquinol they $\left(R_{\mathrm{O}-\mathrm{H} \cdots \mathrm{N}}=2.850\right)$ are close to those reported in cloxiquine and much longer than those reported in 8-quinolinol $\left(R_{\mathrm{O}-\mathrm{H} \cdots \mathrm{N}}=2.793 \AA\right)$. The intramolecular hydrogen bonds in clioquinol and cloxiquine are much more nonlinear $\left[<\mathrm{OHN}=87^{\circ}\right.$ (form Ia) or $95^{\circ}$ (form Ib) and $108^{\circ}$ (form I) or $115^{\circ}$ (form II) for clioquinol and cloxiquine, respectively, versus $109^{\circ}$ in 8-quinolinol] than the intermolecular ones $\left(\angle \mathrm{OHN}=151.5^{\circ}\right.$ and $151.8^{\circ}$ versus $146.1^{\circ}$ and $127.5^{\circ}$ versus $143.0^{\circ}$ in 8 -quinolinol).

The hydrogen bonds were characterized within the Bader QTAIM theory, with their molecular topology described in terms of the BCP and RCP. The topological parameters (bond length $r$, electron density $\rho$, its Laplacian $\Delta \rho$, ellipiticity $\varepsilon$, BCP and RCP) are collected in Tables 3 and 4. They describe molecular stability and characterize the internuclear pathways, which can be classified as shared or closed-shell. All of the expected BCPs associated with the standard covalent bonds and RCPs at the centroids of all benzenoid rings were found in monomers and dimers of both compounds. In addition, in the monomer, one extra BCP was assigned to a weak intramolecular interaction, which in turn generated one more RCP ring, but only in cloxiquine form II. The presence of the RCP and BCP in the monomer of form II of cloxiquine confirms the existence of intramolecular $\mathrm{O}-\mathrm{H} \cdots \mathrm{N}$ hydrogen bonding, since the topological criteria proposed by Koch and Popelier [48] are fulfilled. Surprisingly, in contrast to form II of cloxiquine, the lack of a BCP between the putative donor and the acceptor in the monomer of form I of cloxiquine or form I of cloquinol implies no $\mathrm{O}-\mathrm{H} \cdots \mathrm{N}$ hydrogen bond. This suggests that, if there is this interaction at all, it is very weak and repulsive rather than attractive. The presence of RCPs in fivemembered hydrogen-bonded chelate rings and the presence of BCPs in both kinds of hydrogen bonds in the supramolecular synthon of form II of cloxiquine, see Tables 3 and 4, confirms the existence of different kinds of H-bonds: intramolecular $\mathrm{O}-\mathrm{H} \cdots \mathrm{N}$ and intermolecular $\mathrm{O}-\mathrm{H} \cdots \mathrm{N}$ and $\mathrm{C}-\mathrm{H} \cdots \mathrm{O}$ in form II of cloxiquine, in contrast to the occurrence of only intermolecular $\mathrm{O}-\mathrm{H} \cdots \mathrm{N}$ in the structures of cloxiquine and clioquinol form I.

For the supramolecular synthons of cloxiquine or clioquinol form $\mathrm{I}$, in a similar manner to the monomer, there is no evidence of intramolecular $\mathrm{H}$-bond $\mathrm{O}-\mathrm{H} \cdots \mathrm{N}$, which suggests that the geometric criteria are insufficient to determine the existence of of hydrogen bonding. Additionally, very weak intermolecular halogen contacts $\mathrm{Cl} \cdots \mathrm{I}(\mathrm{Cl} \cdots \mathrm{I}$ $=3.710 \AA)$ and hydrogen bonds $\mathrm{C}-\mathrm{H} \cdots \mathrm{I}\left(R_{\mathrm{C}-\mathrm{H} \cdots \mathrm{I}}=4.026 \AA\right)$ were revealed in the structure of clioquinol, but only in form Ib. The QTAIM calculations yielded electron densities of 0.019 a.u. for intramolecular bonds and $0.020-0.034$ a.u. for intermolecular ones (the electron density falls within a certain range of values, typically between 0.001 and 0.035 a.u.), which are markedly lower than those for the covalent bonds. The corresponding Laplacian values, $\Delta \rho$, are positive and amount to 0.08 a.u. and $0.04-0.09$ a.u. (typically between 0.006 and 0.130 a.u.), which is indicative of the closed-shell interaction. The relief maps of the Laplacian of electron density for clioquinol and cloxiquine in the plane of the intermolecular H-bond O$\mathrm{H} \cdots \mathrm{N}$, see Fig. $8 \mathrm{a}-\mathrm{c}$, exhibit a maximum in the negative Laplacian on either side of the oxygen and nitrogen atoms, corresponding to the lone pair model. Moreover, they show the polarization of nitrogen's lone-pair electrons toward hydrogen and differences in the polarization of the oxygen and nitrogen lone pairs caused by changes in planarity as well as iodine substitution.

To get further insight into the nature of intermolecular and intramolecular interactions, the electronic energy density $H_{\mathrm{BCP}}$ and its components-the local one-electron kinetic energy density $\left(G_{\mathrm{BCP}}\right)$ and the local potential energy density $\left(V_{\mathrm{BCP}}\right)$-were calculated for the charge distribution at the BCP. The hydrogen and halogen bond energies were calculated using $\mathrm{CP}$ as well as the Espinosa method; see Tables 3 and 4. According to Roza's [49] criterion, the intramolecular $\mathrm{O}-\mathrm{H} \cdots \mathrm{N}$ bonds in form II of cloxiquine are weak but slightly stronger than the intermolecular $\mathrm{O}-\mathrm{H} \cdots \mathrm{N}$ bonds (see Tables 3 and 4), but they are generally weaker than typical and mainly electrostatic, while the intermolecular $\mathrm{O}-\mathrm{H} \cdots \mathrm{N}$ bonds in clioquinol and cloxiquine form $\mathrm{I}$ at RT are moderate and partially covalent in nature. The estimated H-bond energies for the systems studied lie within the range $8.33-34.39 \mathrm{~kJ} \mathrm{~mol}^{-1}$; see Tables 3 and 4 . Partitioning the DFT energy of the hydrogen bond into classical components showed that about $75 \%$ was electrostatic (coulombic), and less than 5\% came from polarization and charge transfer.

The large differences in the strengths of the corresponding H-bonds in both forms of cloxiquine suggest that there is interplay between the different $\mathrm{H}$-bonds in adjacent molecules that lead to dimers. It is worth noting that the energies of the H-bonds in form I of cloxiquine estimated by the Espinosa method seem significantly higher at $90 \mathrm{~K}$ than at RT (about $5.6 \mathrm{~kJ} \mathrm{~mol}^{-1}$ ) when the nonoptimized structures are taken into account. Moreover, additional weak intermolecular contact between nitrogen atoms is detected, but only when an extra short $\mathrm{OH}$ bond taken from the X-ray data at 


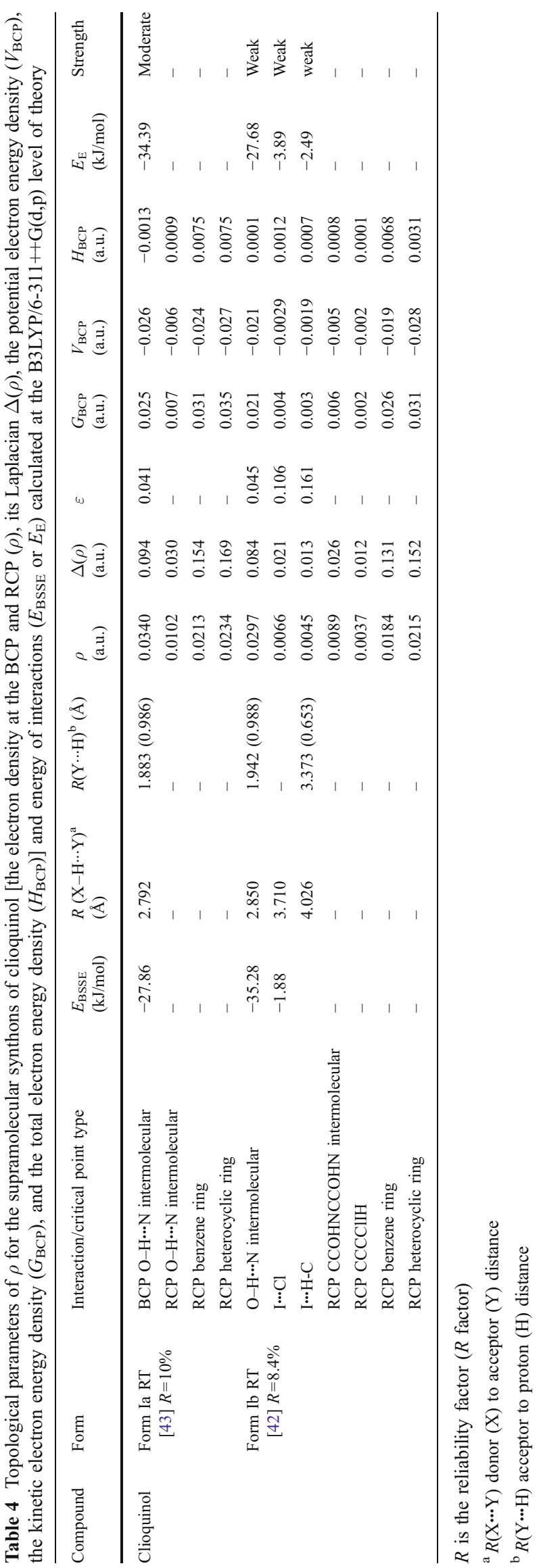

RT is assumed. On the other hand, the hydrogen bond energies for clioquinol forms Ia and Ib differ by as much as $6.5 \mathrm{~kJ} \mathrm{~mol}^{-1}$, but both structures were determined at $\mathrm{RT}$, and the difference in the relevant $R$ factors is only $1.6 \%$. It should be mentioned that the H-bond energy should only be estimated using the Espinosa method when comparing the strengths of H-bonds; it should not be used quantitatively.

According to the QTAIM results, the intermolecular hydrogen bonds $\mathrm{O}-\mathrm{H} \cdots \mathrm{N}$ in cloxiquine and clioquinol (form I) are the strongest interactions, which is in agreement with previous suggestions based on differences in hydrogen acceptor distances [50] as well as IR spectra in solution [51]. In clioquinol, an additional electronwithdrawing substituent (iodine) ortho to the hydroxyl group compeats with chlorine at the para position, resulting in the formation of stronger hydrogen bonds than those in cloxiquine, which is consistent with the IR spectra for the solid (KBr). The direction of the spectral shifts of the $\mathrm{O}-\mathrm{H}$ stretch mode in the mid-infrared, often used to infer the strength of a hydrogen bond, is in a good agreement with the DFT results; see Tables 3 and 4. Proton donor and acceptor sites in hydrogen bonds in supramolecular synthons can be reliably characterized for the solid using ${ }^{1} \mathrm{H}-{ }^{17} \mathrm{O}$ and ${ }^{1} \mathrm{H}_{-}{ }^{14} \mathrm{~N}$ NQDR spectroscopies. It should be noted that the calculations reveal that the presence of the intermolecular $\mathrm{O}-\mathrm{H} \cdots \mathrm{N}$ hydrogen bonding influences $\mathrm{NQR}$ parameters differently at different sites: only slightly for ${ }^{35} \mathrm{Cl}$ (i.e., at the site distant from the hydrogen bond), while significantly at the ${ }^{17} \mathrm{O}$ and ${ }^{14} \mathrm{~N}$ participating in this bond; a comparison of the experimental data and the results of calculations for the monomer and stacked dimers (see Tables 3 and 4) provides a drastic example.

The presence of the electron-withdrawing iodine substituent at position 7 of 5-chloro-8-quinolinol results in a decrease in the proton affinity of the nitrogen atom and an increase in the hydrogen bond strength, which induces changes in the values of $e^{2} Q q h^{-1}$ and $\eta$; see Tables 1 and 2 .

As a result of this substitution, $e^{2} Q q h^{-1}$ increases on ${ }^{35} \mathrm{Cl}$ and ${ }^{14} \mathrm{~N}$ by 0.01 and $2 \%$, respectively, while on ${ }^{17} \mathrm{O}$ this parameter is predicted to decrease by $0.05 \%$. The predicted changes in $\eta$ on ${ }^{35} \mathrm{Cl}$ are negligibly small, on ${ }^{17} \mathrm{O}$ this parameter decreases by $3 \%$, but on ${ }^{14} \mathrm{~N}$ it increases by $2 \%$. The direction of the changes in the NQR parameters observed upon switching from cloxiquine to clioquinol is in agreement with Seliger's [52] observation that when shortening and hence also strengthening the hydrogen bond, the asymmetry parameter $\eta$ increases. A considerable increase in $e^{2} Q q h^{-1}$ on the chlorine atom, which implies a decrease in the symmetry of the charge distribution at this atom (i.e., nonspherical symmetry), is also observed mainly as a consequence of the presence of the iodine electronwithdrawing substituent meta to the chlorine. A comparison 
Fig. 6 The orientations of the EFG tensor axes at all quadrupolar sites: cloxiquine (form I), first column; cloxiquine (form II), second column; clioquinol (form Ia), third column. In each case, one of the axes is perpendicular to the screen

Fig. 7 Molecular graphs of 8quinolinol derivatives: cloxiquine (form I), supramolecular synthon; cloxiquine (form II), supramolecular synthon; clioquinol (form Ia), supramolecular synthon. Dashed lines indicate the interactions, large circles correspond to attractors, and small circles to critical points (red RCP, green $\mathrm{BCP})$
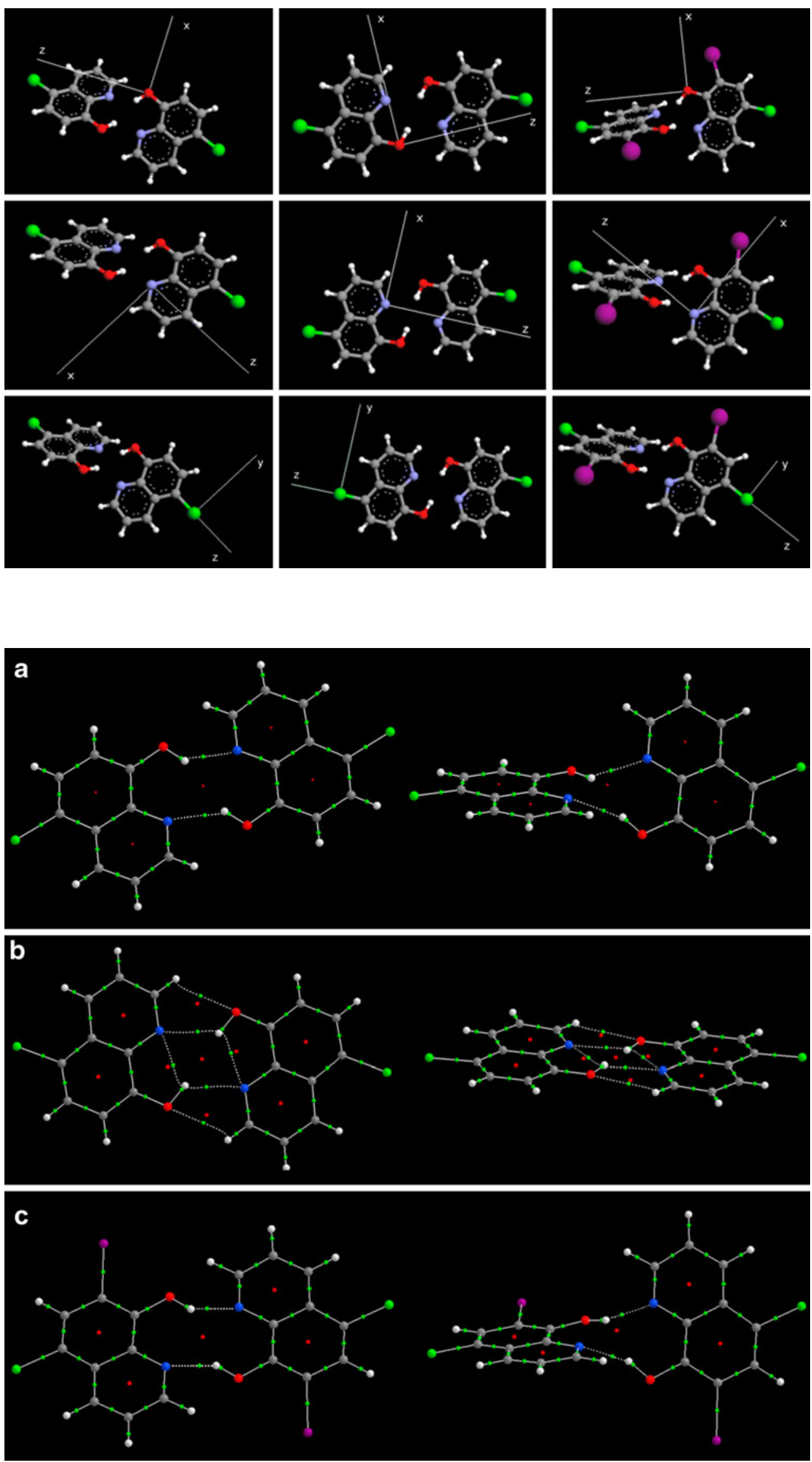
Fig. 8 Relief map of the Laplacian of electron density in the $\mathrm{OH}^{\cdots} \mathrm{N}$ plane: cloxiquine (form I), supramolecular synthon; cloxiquine (form II), supramolecular synthon; clioquinol (form Ia), supramolecular synthon
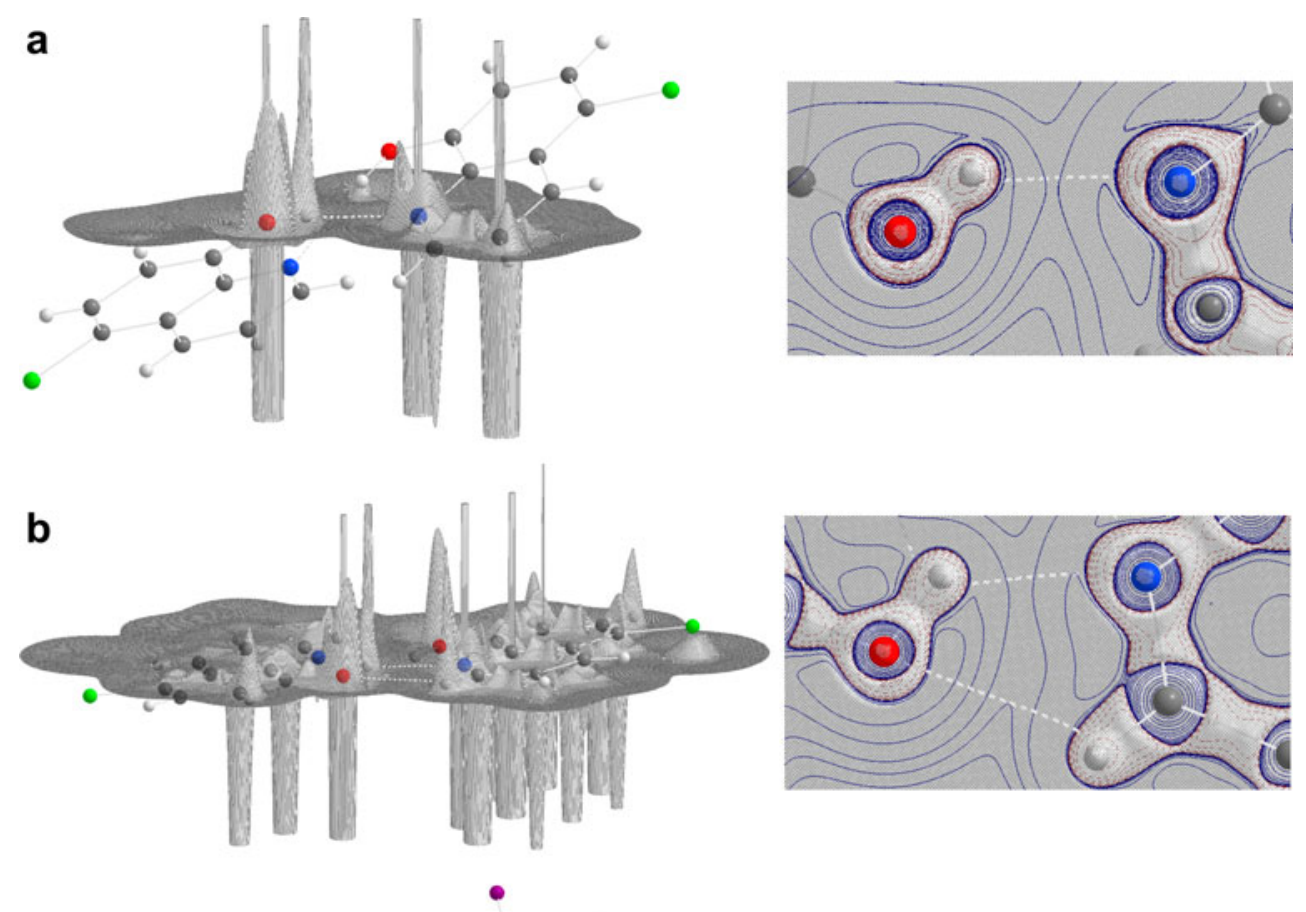

C
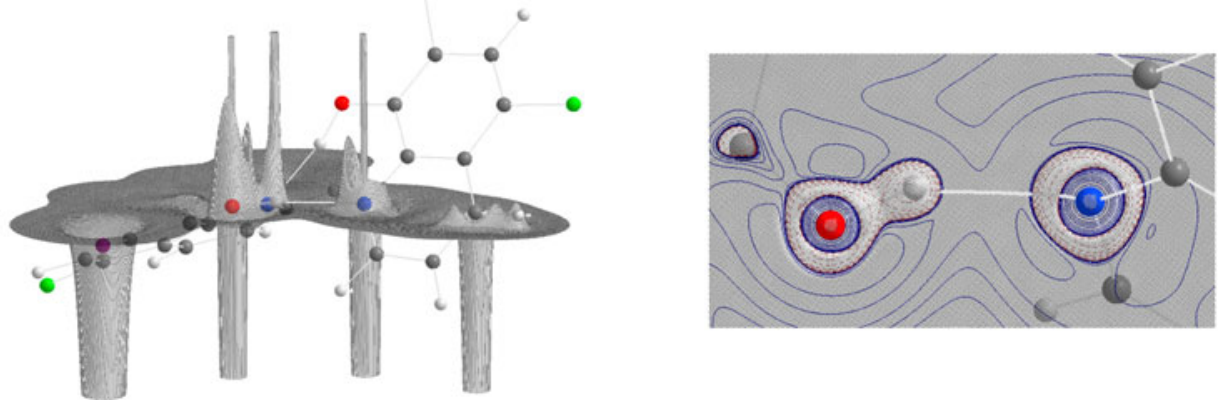

of the results obtained for clusters of different sizes (see Table 2) shows that the value of $e^{2} Q q h^{-1}$ depends strongly on the presence of hydrogen bonding, and that $\eta$ can be used as an indicator of the strength of the hydrogen bonding.

Because of the relatively short distances to the protons (2.182 and $2.622 \AA)$ in the structures of cloxiquine and clioquinol, $\mathrm{O}-\mathrm{H} \cdots \mathrm{H}-\mathrm{O}$ dihydrogen bonds could be expected as well as $\mathrm{HB}$ intermolecular interactions. An $\mathrm{H} \cdots \mathrm{H}$ distance of less than $2.4 \AA$ [i.e., twice the van der Waals radius of the hydrogen atom $(1.2 \AA)]$ is the geometrical criterion that is most widely used to identify the formation of this type of hydrogen bond. However, because the dihydrogen bonds are electrostatic in nature, and such interactions act beyond this distance, this van der Waals criterion has been strongly criticized [53]. Additional criteria taken into account include the interaction energy (which falls within the same range that are typical of hydrogen bonds: $12-41 \mathrm{~kJ} \mathrm{~mol}^{-1}$ ), the linearity of the dihydrogen bonds, and the difference between the charges on both of the electronegative atoms [54, 55]. QTAIM does not detect the critical points that indicate the presence of an $\mathrm{O}-\mathrm{H} \cdots \mathrm{H}-\mathrm{O}$ dihydrogen bond for any form of clioquinol or cloxiquine, which appears to be due to both oxygen atoms having the same polarization, as a consequence of the symmetric dimeric structure.

\section{Stacked supermolecular synthon}

An important type of interaction that is specific to the solid state and commonly seen in aromatic systems is the vertical stacking of parallel supramolecular synthons; see Fig. 9a-c. As mentioned earlier, in the dimeric structures of clioquinol and cloxiquine (form I), the paired molecules in the units are twisted and the units expand into columns linked by $\pi-\pi$ stacking interactions with distinct layers separated by only $3.814 \AA$ in cloxiquine [45] (i.e., close to the $3.811 \AA$ observed in 8-quinolinol [46]), and as large as 4.141 or $4.161 \AA$ in clioquinol $[42,43]$, which suggests that there is a great deal of steric repulsion from I-I, forcing the large spacing between layers. The difference between forms I and II of cloxiquine (Fig. 9a, b) is that the twisted and 
Fig. 9 Molecular graphs of 8quinolinol derivatives: cloxiquine (form I), stacked supramolecular synthon; cloxiquine (form II), stacked supramolecular synthon; clioquinol (form Ia), stacked supramolecular synthon; dashed lines indicate interactions, large circles correspond to attractors, small circles to critical points (green $\mathrm{BCP}$; RCPs are omitted for clarity)
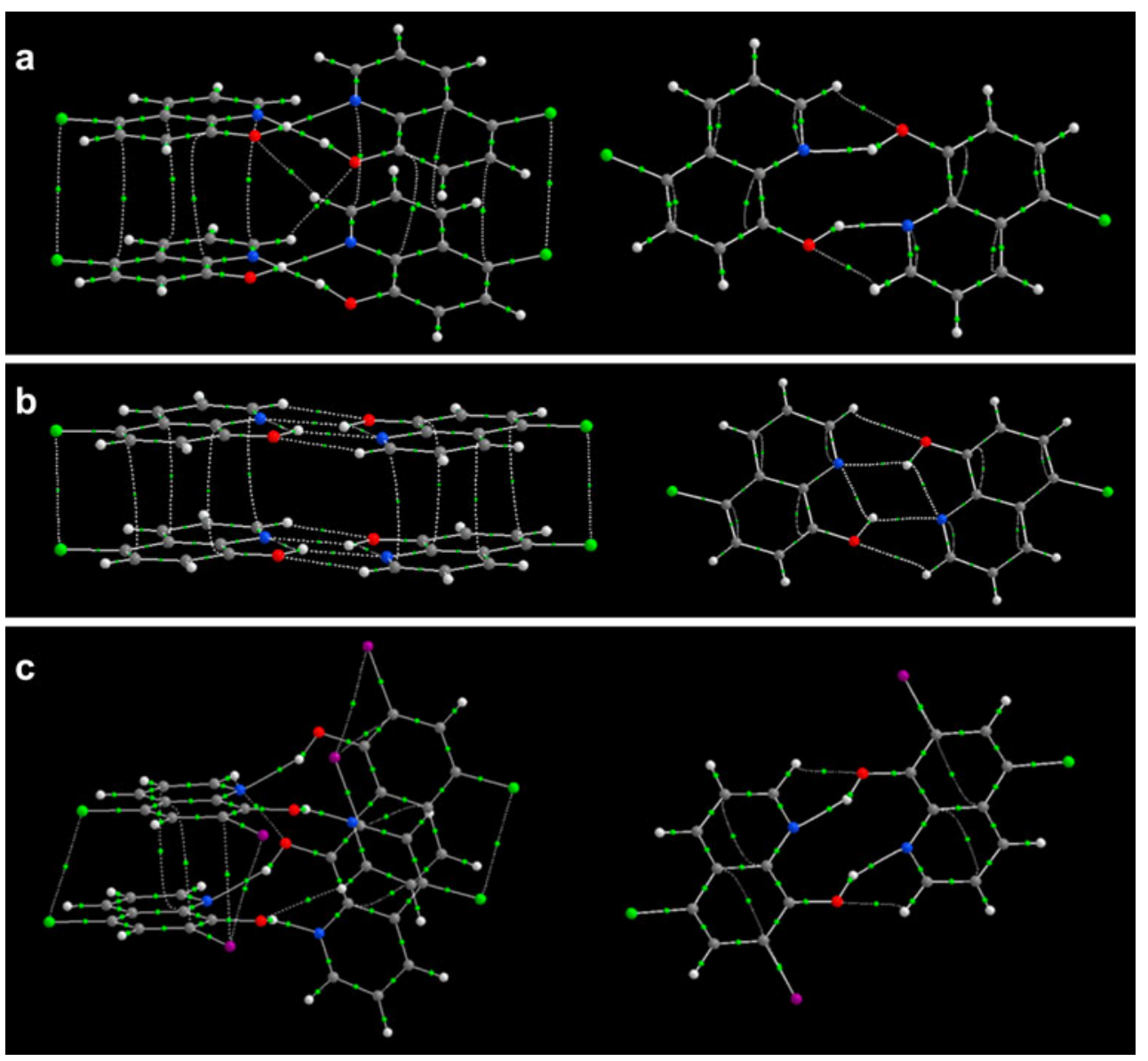

nontwisted adjacent supramolecular synthons form columns along different crystallographic axes ( $\mathrm{c}$ in form $\mathrm{I}$ and $\mathrm{b}$ in form II) [45]. The $\pi-\pi$ stacking interactions between adjacent supramolecular synthons (stacked dimers) enhance the stability of the crystal structure in both clioquinol and cloxiquine, irrespective of the specific polymorph; however, their strengths are expected to differ because of large differences in the polarizations of the adjacent molecules, so dimers are also responsible for the specific arrangements seen in parallel columns.

The dipole moments calculated by the DFT method for a continuous distribution of electron density provide essential information on the overall polarity of the charge system. The monomer of clioquinol has a higher dipole moment than that of form I of cloxiquine (Ia: 3.15 ; Ib: 3.20 versus $1.71 \mathrm{D}$ for form I, RT), while the dimer and stacked dimers have lower dipole moments (for form Ia of clioquinol: 3.97; form Ib: 3.94 versus $1.97 \mathrm{D}$ for form I of cloxiquine at RT, and form Ia of clioquinol: 3.07; form $\mathrm{Ib}: 3.03$ versus $4.14 \mathrm{D}$ for form I of cloxiquine at RT). Moreover, in form II of cloxiquine, which is predicted to be energetically more stable than form I (by $13.6 \mathrm{~kJ} \mathrm{~mol}^{-1}$ ), the monomer has a slightly higher dipole moment $(1.82 \mathrm{D})$, while the dimer and stacked dimers are nonpolar $(0.02$ and $0.005 \mathrm{D}$, respectively), which suggest large differences in the polarizations of the adjacent molecules, so that the dimers are closely connected with the specific arrangement. The stacking found in both clioquinol and cloxiquine (form I) involves similar overlapping, albeit much smaller than observed in cloxiquine (form II). The nucleus-independent chemical shift (NICS) calculated at the geometrical center, and the modified form of this parameter, NICS(1), calculated at $1 \AA$ above the plane of the ring, both reflect $\pi$ effects and are both negative, which means that the criterion of Schleyer et al. [56] for aromaticity is fulfilled for both compounds irrespective of the form. The standard method of estimating the energy of stacking $\pi \cdots \pi$ interactions (CP) appears to be ineffective for such a complicated system. The roughly estimated energies of $\pi-\pi$ stacking interactions are as high as 16.3 and $14.5 \mathrm{~kJ}$ $\mathrm{mol}^{-1}$ for clioquinol forms Ia and Ib, respectively, and 19.8 and $23.6 \mathrm{~kJ} \mathrm{~mol}^{-1}$ for cloxiquine forms I and II, respectively. A comparison of these energies suggests that the strength of the stacking $\pi \cdots \pi$ interaction depends on the planarity (i.e., it is much weaker in form I than in form II of cloxiquine), and that the lower energy of $\pi-\pi$ stacking for clioquinol in comparison to cloxiquine is due to the greater spacing between layers. 


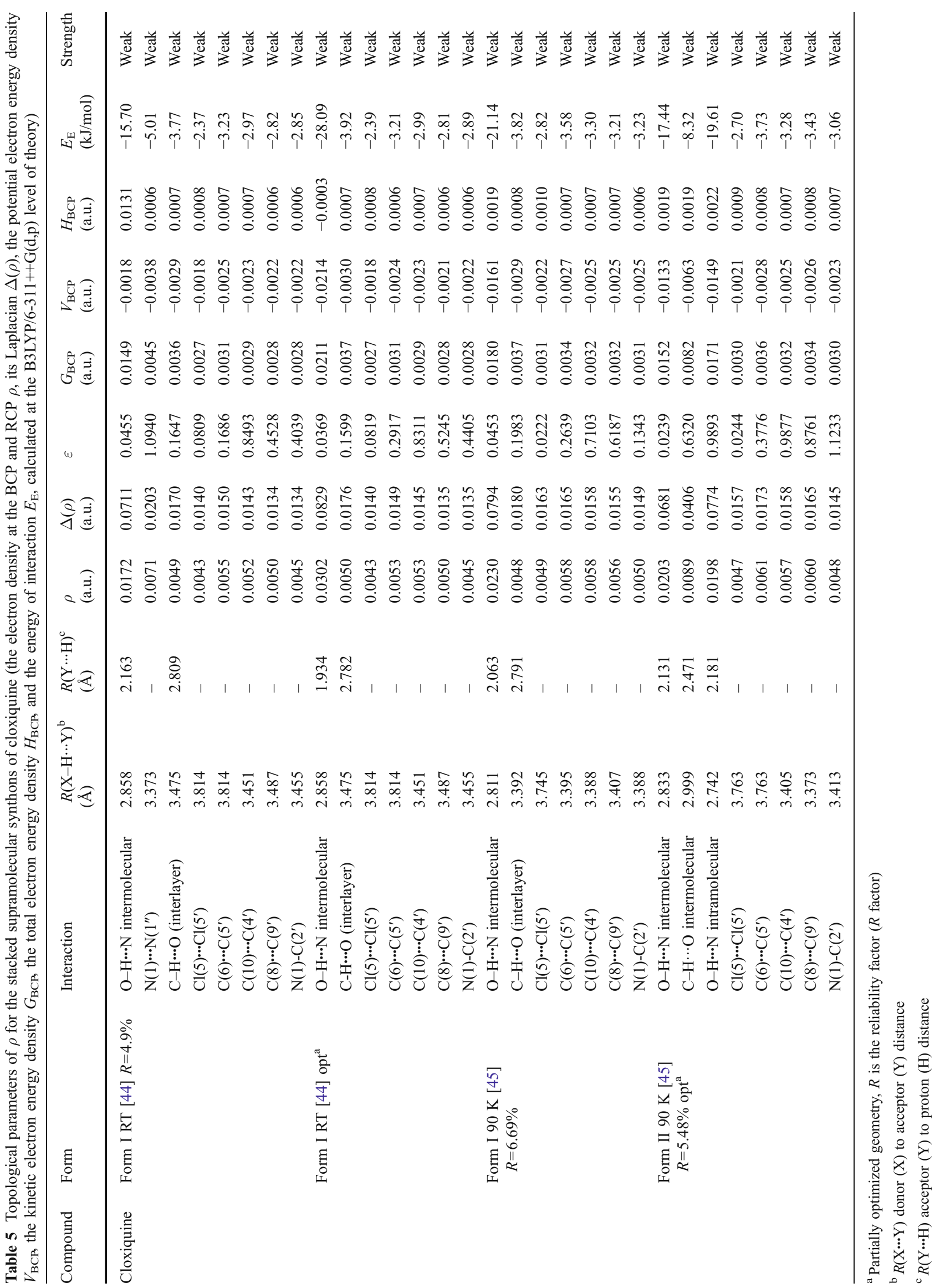




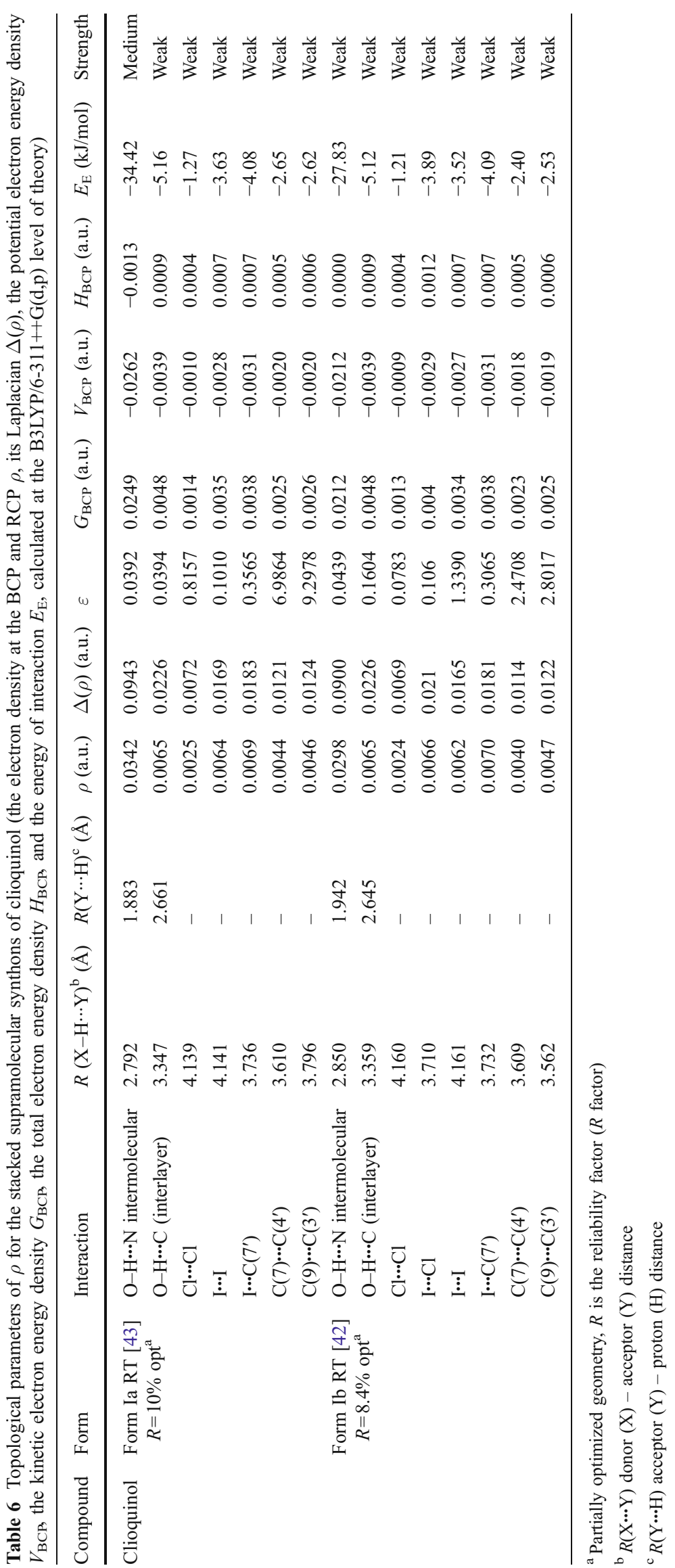


A more detailed examination of the closest-neighbor stacked supramolecular synthons revealed one intermolecular hydrogen bond $\mathrm{C}-\mathrm{H} \cdots \mathrm{O}$ (only form I of cloxiquine, but both forms of clioquinol) and five unique intermolecular atomic contacts (all forms), in addition to the intermolecular bonds revealed earlier in the crystal packing of both compounds; see Fig. 9. According to the topological parameters reported in Tables 5 and 6 , all of the intermolecular interactions analyzed here are weak except for the intermolecular $\mathrm{O}-\mathrm{H} \cdots \mathrm{N}$ hydrogen bonds. The topology of these weak intermolecular interactions, which are characterized by very small values of $\rho(r)$, small positive values of the Laplacian, high values of $\varepsilon$, values of $H_{\mathrm{BCP}}$ close to zero, and values of $\left|V_{\mathrm{BCP}}\right| / G_{\mathrm{BCP}} \leq 1$ (see Tables 5 and 6), was analyzed using QTAIM. In terms of the Espinosa classification [41], these interactions fall between the pure closed-shell and transit types. The energies of these weak interactions depend linearly on the electron density at the critical point and the Laplacian of the density (see Fig. 10a, b). The high values of ellipticity are linked to the relatively low value of the second Hessian eigenvalue, and thus the presence of an RCP as well as a $\mathrm{BCP}$ cannot be ascribed to $\pi$-bonding.

According to a comparative analysis of the BCPs and RCPs of forms I and II of cloxiquine, there is one hydrogen bond $\mathrm{O}-\mathrm{H} \cdots \mathrm{N}$ in form $\mathrm{I}$, while there are two competing hydrogen bonds $\mathrm{O}-\mathrm{H} \cdots \mathrm{N}$ (intra- and intermolecular; the former is somewhat stronger than the latter) in form II (see Tables 5 and 6). Moreover, the intermolecular $\mathrm{C}-\mathrm{H} \cdots \mathrm{O}$ in form I links the molecules from the same supramolecular synthon (see Fig. 9a), while in form II it links the molecules from different (stacked) supramolecular synthons (see Fig. 9b) and is much weaker (almost half the strength). Such a pattern of bonds induces planarity in the supramolecular synthons in form II of cloxiquine, and it is also responsible for the difference in the polarities of the supramolecular synthons for forms I and II of cloxiquine. The ellipticities of all of the covalent bonds in which nitrogen and oxygen atoms participate do not change significantly in either form of cloxiquine. Hence, we may conclude that the planarity does not force $\pi$-electron delocalization within the quinolinol ring. The large change in ellipticity at the BCP of the $\mathrm{N} \cdots \mathrm{O}$ bond suggests that the planarity forces $\pi$-electron delocalization in the pseudo decamembered ring. The H-bond energies listed in Tables 3-6 (i.e., for all supramolecular synthons and stacked supramolecular synthons) are very well correlated (see Fig. 10c), and the differences between them do not exceed $0.19 \mathrm{~kJ} \mathrm{~mol}^{-1}$, which means that there is no interplay between the $\mathrm{H}$-bonds in adjacent molecules that create supramolecular synthons and the intermolecular interactions between stacked supramolecular synthons. Additionally, Fig. 11a illustrates that there is a strong
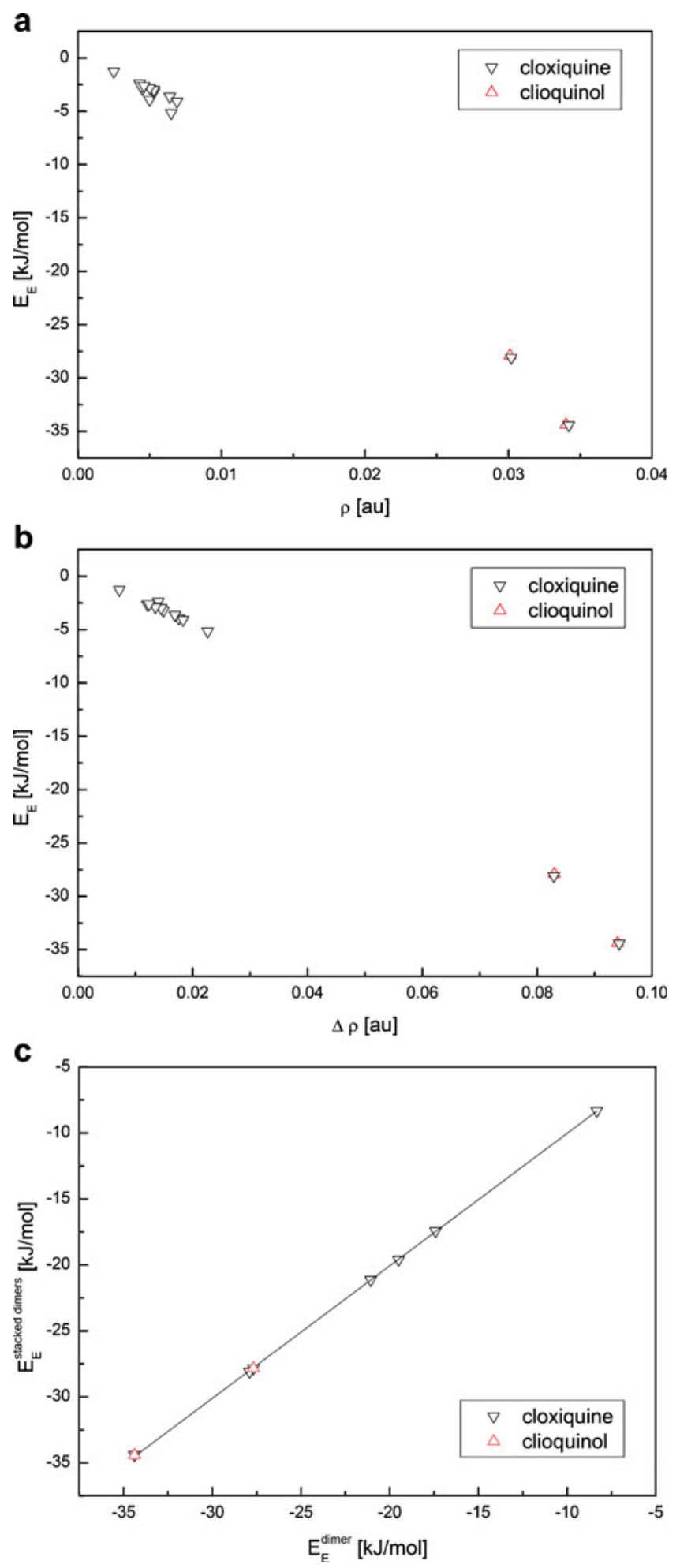

Fig. 10a-c The relations between a $\rho$ (the electron density at the critical point) and the interaction energy (in $\mathrm{kJmol}^{-1}$ ), b $\Delta \rho$ (the Laplacian of electron density at the critical point) and the interaction energy (in $\mathrm{kJmol}^{-1}$ ), and $\mathbf{c}$ the energy of hydrogen bond interactions $E_{\mathrm{E}}\left(\right.$ in $\mathrm{kJ} \mathrm{mol}^{-1}$ ) for stacked dimers, and the corresponding energy for dimers, calculated using the Espinosa method assuming dimer and stacked dimer formation (solid line linear fit, correlation coefficient 0.999 , standard deviation $0.02 \mathrm{kJmol}^{-1}$ and slope $1.004 \mathrm{kJmol}^{-1}$ ) 
Fig. 11a-c The relations between a $\mathrm{H} \cdots \mathrm{Y}$ distance $\left(R_{\mathrm{H} \cdots \mathrm{Y}}\right)$ and hydrogen bond energy (in $\mathrm{kJ} \mathrm{mol}^{-1}$ ); solid line fit with a parabola, correlation coefficient 0.989 , standard deviation $11.9 \mathrm{~kJ} \mathrm{~mol}^{-1}$, b H. $\cdots \mathrm{Y}$ distance $\left(R_{\mathrm{H} \cdots \mathrm{Y}}\right)$ and $\rho$ (electron density at the critical point); solid line fit with an exponential function, correlation coefficient 0.998 , standard deviation $2.4 \times 10^{-6}$ a.u., and $\mathbf{c}$ the $\mathrm{H} \cdots \mathrm{Y}$ distance $\left(R_{\mathrm{H} \cdots \mathrm{Y}}\right)$ and $\Delta \rho$ (Laplacian of the electron density at the critical point); solid line fit with a linear function correlation coefficient 0.988 , standard deviation $1.2 \times 10^{-4}$ a.u., slope -0.085 a.u.

parabolic relationship between the $\mathrm{H} \cdots \mathrm{Y}$ distance and the estimated hydrogen bond energy $E_{\mathrm{E}}$. The electron density and the Laplacian of the density at the BCP of the $\mathrm{H} \cdots \mathrm{Y}$ contact depend exponentially and linearly on the $\mathrm{H} \cdots \mathrm{Y}$ distance, respectively (see Fig. $11 \mathrm{~b}$ and c). It is worth noting that the points in Figs. 10 and 11 for dimer and stacked dimer overlap, so only one set (the second case) is shown, which confirms the lack of interplay between the Hbonds in adjacent molecules that form dimers and intermolecular interactions between stacked dimers. It should be emphasized that these dependencies that characterize $\mathrm{H}$ bonds in stacked supramolecular synthons (see Figs. 10c and $11 \mathrm{a}-\mathrm{c})$ are in excellent agreement with the results previously reported by Espinosa but which were obtained for small molecular systems [41].

The pattern of the intra- and intermolecular interactions in clioquinol form I (Ia and Ib) is generally similar to that observed for cloxiquine form I (see Tables 5 and 6, Figs. 7, $8,9,10$, and 11), but the smaller number of weak interactions between the carbon atoms from heterocyclic rings (two versus four) and the appearance of two (form Ia) or three (form $\mathrm{Ib}$ ) weak interactions from I contacts should be indicated. The ellipticities of all (covalent and hydrogen) bonds in which nitrogen and oxygen atoms participate increase significantly in clioquinol in comparison to cloxiquine. Hence, we may conclude that the electronwithdrawing substituent influences $\pi$-electron delocalization within the quinolinol ring and pseudo decamembered ring. According to our experimental and theoretical results, the oxygen atom is much more sensitive to differences in supramolecular synthon planarity than the nitrogen atom involved in the same $\mathrm{O}-\mathrm{H} \cdots \mathrm{N}$ bond. The considerable differences in the NQR parameters for the planar and twisted supramolecular synthons permit differentiation between the specific polymorphic forms and suggest the presence of form I in both experimentally studied samples.

\section{Conclusions}

1. The NQR parameters $e^{2} Q q h^{-1}$ and $\eta$ at the $\mathrm{O}$ site indicate differences in supramolecular synthon planarity and thus enable differentiation between the polymorphic forms. This conclusion is based on the much

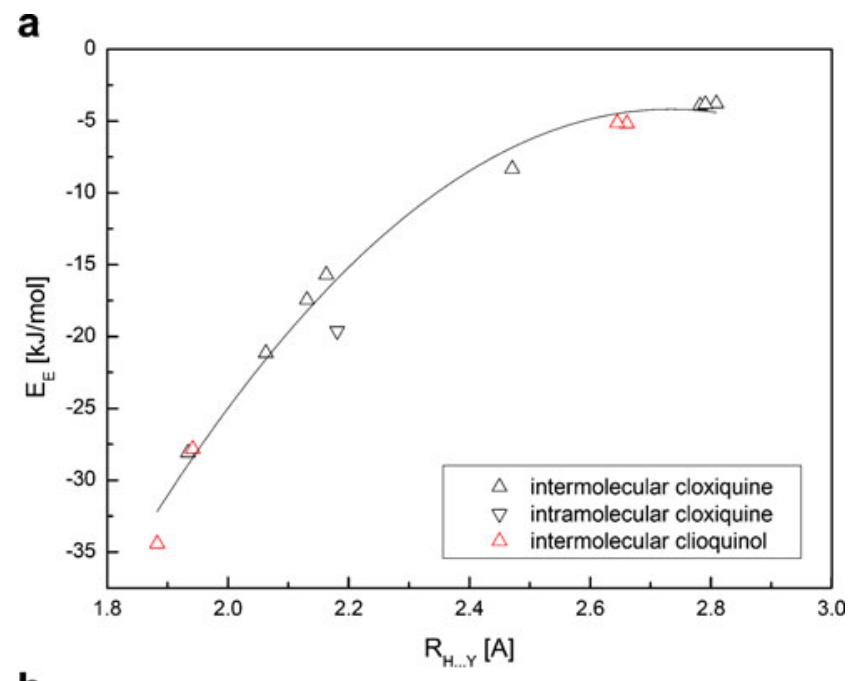

b

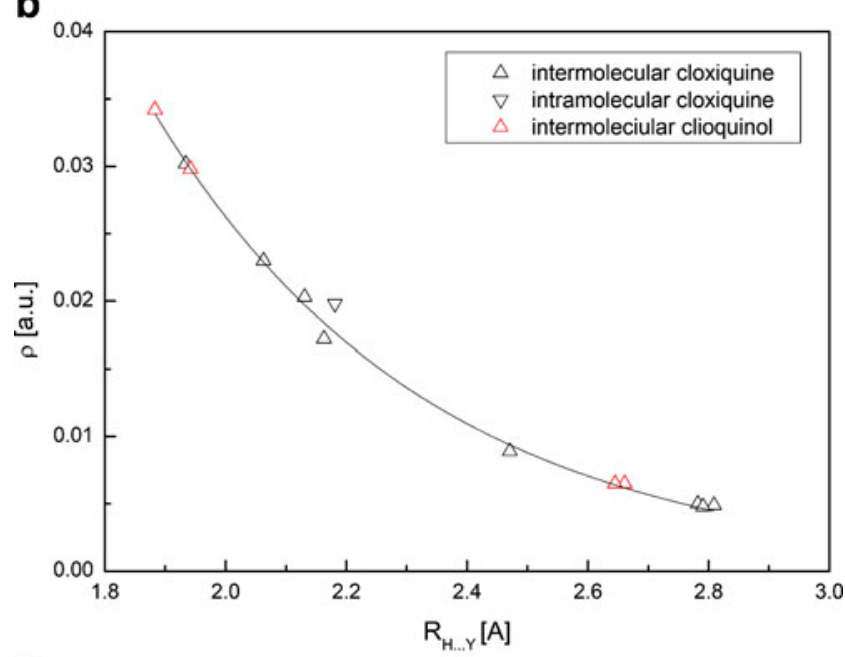

C

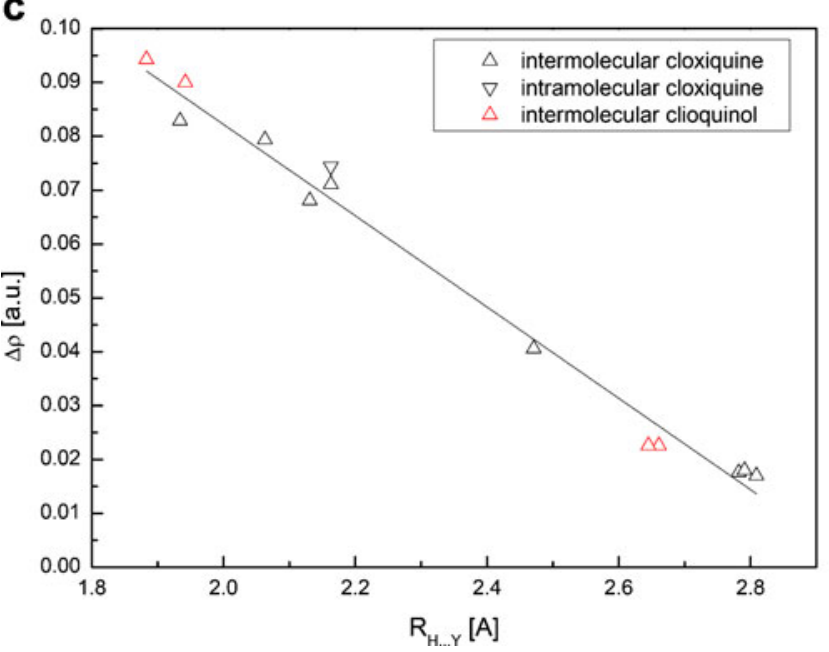

better correlation between experimental and DFTcalculated NQR frequencies for form I than for form II.

2. The results of QTAIM analysis suggest interplay between the different $\mathrm{H}$-bonds in adjacent molecules that form dimers, but no interplay between the H-bonds 
in adjacent molecules that form dimers and the weak intermolecular interactions between stacked dimers.

3. The pattern of the intra- and intermolecular interactions in forms Ia and $\mathrm{Ib}$ of clioquinol is generally similar to that observed for form I of cloxiquine. The presence of the I (iodine) substituent at the $\mathrm{C}(7)$ position is crucial because this substituent enables clioquinol to form stronger hydrogen bonds and reduce the number of weak interactions between the carbon atoms from heterocyclic rings. Moreover, the electron-withdrawing substituent (iodine) influences $\pi$-electron delocalization within the quinolinol ring and the pseudo decamembered ring, which can be easily observed as a change in the NQR parameters at $\mathrm{N}$ and $\mathrm{O}$ atoms.

4. A comparison of the results for monomers, dimers and clusters (stacked dimers) shows a systematic improvement in the reproduction of the NQR parameters to a degree that is proportional to the strength of the interactions (the weaker the interaction, the smaller the correction).

Acknowledgments A generous allotment of computer time from the PCSS (Poznań Supercomputing and Networking Centre) in Poland is gratefully acknowledged.

Open Access This article is distributed under the terms of the Creative Commons Attribution Noncommercial License which permits any noncommercial use, distribution, and reproduction in any medium, provided the original author(s) and source are credited.

\section{References}

1. Rohde W, Mikelens P, Jackson J, Blackman J, Whitcher J, Levinson W (1976) Antimicrob Agents Chemother 10:234240

2. Fischer T, Fagerlund C, Hartvig P (1978) Acta Derm Venereol 58:407-411

3. Gholz LM, Arons WL (1964) Am J Trop Med Hyg 13:396-401

4. Krogh CME (ed)(1991) Compendium of pharmaceuticals and specialties. Canadian Pharmaceutical Association, Ottawa, p 1311

5. Medical Economics Company (1991) Physicians' desk reference. Medical Economics Company, Oradell, p 874

6. Burger A (1970) Medicinal chemistry, 3rd edn. Wiley, New York

7. Yassin MS, Ekblom J, Xilinas M (2000) J Neurol Sci 173:4044

8. Kaiser HJ, Kunze J (1982) Zubereitungen Fortschr Ther 100:1265-1267

9. Hongmanee P, Rukseree K, Buabut B, Somsri B, Palittapongarnpim P (2007) Antimicrob Agents Chemother 51:1105-1106

10. Thomas PK, Bradley DJ, Bradley WA, Degen PH, Krinke G, Muddle J, Schaumburg HH, Skeltonstroud PN, Thomann P, Tzebelikos E (1984) J Neurol Sci 64:277-295

11. Tjälve H (1984) Med Hypotheses 15:293-299

12. Baumgartner G, Gawel HE, Pallis CA, Clifford F, Schaumburg HH, Thomas PK, Wadia NH (1979) J Neurol Neurosurg Psychiatry 42:1075-1083

13. Ricoy RJ, Ortega A, Cabello A (1982) J Neurol Sci 53:241-251
14. Nguyen T, Hamby A, Massa SM (2005) Proc Natl Acad Sci USA 102:11840-11845

15. Ritchie CW, Bush AI, Masters CL (2004) Expert Opin Investig Drugs 13:1585-1592

16. Cherny RA, Atwood CS, Xilinas ME, Gray DN, Jones WD, McLean CA, Barnham KJ, Volitakis I, Fraser FW, Kim Y, Huang X, Goldstein LE, Moir RD, Lim JT, Beyreuther K, Zheng H, Tanzi RE, Masters CL, Bush AI (2001) Neuron 30:665-676

17. Ritchie CW, Bush AI, Mackinnon A, Macfarlane S, Mastwyk M, MacGregor L, Kiers L, Cherny R, Li QX, Tammer A, Carrington D, Mavros C, Volitakis I, Xilinas M, Ames D, Davis S, Beyreuther K, Tanzi RE, Masters CL (2003) Arch Neurol 60:1685-1691

18. Wang Y, Branicky R, Stepanyan Z, Carroll M, Guimond MP, Hihi A, Hayes S, Mcbride K, Hekimi S (2009) J Biol Chem 284:314-323

19. Mao X, Li SX, Sprangers R, Wang X, Venugopal A, Wood T, Zhang Y, Kuntz DA, Coe E, Trudel S, Rose D, Batey RA, Kay LE, Schimmer AD (2009) Leukemia 23:585-590

20. Ding WQ, Liu B, Vaught JL, Yamauchi H, Lind SE (2005) Cancer Res 65:3389-3395

21. Latosińska JN (2005) J Mol Graph Model 23:329-337

22. Latosińska JN (2007) Expert Opin Drug Discov 2:225-248

23. Latosińska JN, Latosińska M, Seliger J, Žagar V, Kazimierczuk Z (2009) Chem Phys Lett 476:293-302

24. Latosińska JN, Seliger J, Žagar V, Burchardt DV (2009) J Phys Chem A 113:8781-8790

25. Latosińska JN, Latosińska M, Seliger J, Žagar V, Maurin JK, Orzeszko A, Kazimierczuk Z (2010) J Phys Chem A 114:563575

26. Seliger J (2000) NQR theory. In: Lindon JC, Tranter GE, Holmes JL (eds) Encyclopedia of spectroscopy and spectrometry. Academic, London, pp 1672-1680

27. Seliger J, Osredkar R, Mali M, Blinc R (1976) J Chem Phys 65:2887-2891

28. Seliger J, Blinc R, Arend H, Kind R (1976) Z Phys B 25:185-189

29. Stephenson D, Smith JAS (1988) Proc R Soc Lond A 416:149178

30. Seliger J, Žagar V (2008) J Magn Reson 193:54-62

31. Seliger J, Žagar V, Blinc R (1994) J Magn Reson A 106:214-222

32. Slusher RE, Hahn EL (1968) Phys Rev 166:332-347

33. Seliger J, Žagar V (2008) J Magn Reson 194:175-181

34. Brosnan SGP, Edmonds DT (1981) J Magn Reson 45:440-450

35. Seliger J, Žagar V, Blinc R, Novak A (1986) J Chem Phys 84:5857-5861

36. Frisch MJ, Trucks GW, Schlegel HB, Scuseria GE, Robb MA, Cheeseman JR, Montgomery JA Jr, Vreven T, Kudin KN, Burant JC, Millam JM, Iyengar SS, Tomasi J, Barone V, Mennucci B, Cossi M, Scalmani G, Rega N, Petersson GA, Nakatsuji H, Hada M, Ehara M, Toyota K, Fukuda R, Hasegawa J, Ishida M, Nakajima T, Honda Y, Kitao O, Nakai H, Klene M, Li X, Knox JE, Hratchian HP, Cross JB, Bakken V, Adamo C, Jaramillo J, Gomperts R, Stratmann RE, Yazyev O, Austin AJ, Cammi R, Pomelli C, Ochterski JW, Ayala PY, Morokuma K, Voth GA, Salvador P, Dannenberg JJ, Zakrzewski VG, Dapprich S, Daniels AD, Strain MC, Farkas O, Malick DK, Rabuck AD, Raghavachari K, Foresman JB, Ortiz JV, Cui Q, Baboul AG, Clifford S, Cioslowski J, Stefanov BB, Liu G, Liashenko A, Piskorz P, Komaromi I, Martin RL, Fox DJ, Keith T, Al-Laham MA, Peng CY, Nanayakkara A, Challacombe M, Gill PMW, Johnson B, Chen W, Wong MW, Gonzalez C, Pople JA (2004) Gaussian 03, revision D01. Gaussian Inc., Wallingford

37. Becke AD (1993) J Chem Phys 98:1372-1377

38. Lee C, Yang W, Parr RG (1988) Phys Rev B 37:785-789

39. Bader RFW (1994) Atoms in molecules: a quantum theory. Oxford University Press, Oxford

40. Boys SB, Bernardi F (1970) Mol Phys 17:553-566 
41. Espinosa E, Molins E, Lecomte C (1998) Chem Phys Lett 285:170-173

42. Kashino S, Haisa M (1973) Bull Chem Soc Jpn 46:10941098

43. Leger JM, Marsau P, Housty J (1972) C R Acad Sci Ser C (Chim) 274:1991

44. Banerjee T, Saha NN (1986) Acta Cryst C 42:1408-1411

45. Ma Z, Moulton B (2009) J Chem Cryst 39:913-918

46. Roychowdhury P, Das BN, Basak BS (1978) Acta Cryst B 34:1047-1048

47. Desiraju GR (1994) Angew Chem Int 34:2311-2327

48. Koch PLA, Popelier A (1995) J Phys Chem 99:9747-9754
49. Rozas I, Alkorta I, Elguero J (2000) J Am Chem Soc 122:1115411161

50. Ceccarelli C, Jeffrey GA, Taylor R (1981) J Mol Struct 70:255-271

51. Badger GM, Moritz AG (1958) J Chem Soc 3437-3442

52. Seliger J (1998) Chem Phys 231:81-86

53. Desiraju GR, Steiner T (1999) The weak hydrogen bond in structural chemistry and biology. Oxford University Press, New York

54. Grabowski SJ (2004) J Phys Chem A 108:5823-5830

55. Grabowski SJ, Sokalski WA, Leszczynski J (2005) J Phys Chem A 109:4331-4341

56. Schleyer PvR, Maercker C, Dransfield A, Jiao H, Hommes NJ, Hommes RE (1996) J Am Chem Soc 118:6317-6318 